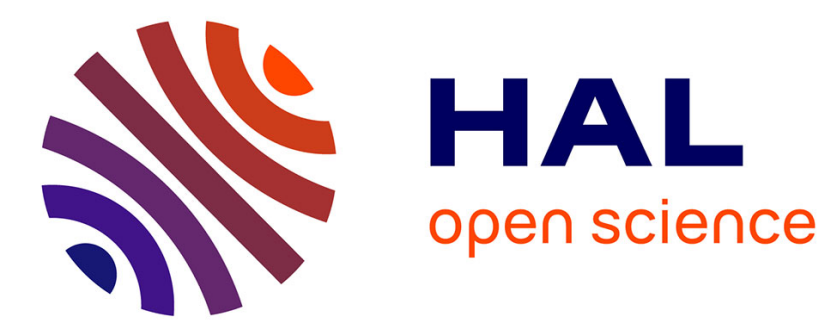

\title{
Equations, Contractions, and Unique Solutions
}

Davide Sangiorgi

\section{To cite this version:}

Davide Sangiorgi. Equations, Contractions, and Unique Solutions. ACM Transactions on Computational Logic, 2017, 18 (1), pp.1-36. 10.1145/2971339 . hal-01647063

\section{HAL Id: hal-01647063 \\ https://hal.inria.fr/hal-01647063}

Submitted on 26 Nov 2017

HAL is a multi-disciplinary open access archive for the deposit and dissemination of scientific research documents, whether they are published or not. The documents may come from teaching and research institutions in France or abroad, or from public or private research centers.
L'archive ouverte pluridisciplinaire HAL, est destinée au dépôt et à la diffusion de documents scientifiques de niveau recherche, publiés ou non, émanant des établissements d'enseignement et de recherche français ou étrangers, des laboratoires publics ou privés. 


\title{
Equations, contractions, and unique solutions*
}

\author{
Davide Sangiorgi \\ University of Bologna and INRIA
}

January 2017

\begin{abstract}
One of the most studied behavioural equivalences is bisimilarity. Its success is much due to the associated bisimulation proof method, which can be further enhanced by means of 'bisimulation up-to' techniques such as 'up-to context'.

A different proof method is discussed, based on unique solution of special forms of inequations called contractions, and inspired by Milner's theorem on unique solution of equations. The method is as powerful as the bisimulation proof method and its 'up-to context' enhancements. The definition of contraction can be transferred onto other behavioural equivalences, possibly contextual and non-coinductive. This enables a coinductive reasoning style on such equivalences, either by applying the method based on unique solution of contractions, or by injecting appropriate contraction preorders into the bisimulation game.

The techniques are illustrated on CCS-like languages; an example dealing with higher-order languages is also shown.
\end{abstract}

\section{Introduction}

Bisimilarity is employed to define behavioural equivalences and reason about them. Originated in concurrency theory, bisimilarity is now widely used also in other areas, as well as outside Computer Science.

In this paper, behavioural equivalences, hence also bisimilarity, are meant to be weak because they abstract from internal moves of terms, as opposed to the strong ones, which make no distinctions between the internal moves and the external ones (i.e., the interactions with the environment). Weak equivalences are, practically, the most relevant ones: e.g., two equal programs may produce the same result with different numbers of evaluation steps.

In proofs of bisimilarity results, the bisimulation proof method has become predominant, particularly with the enhancements of the method provided by the so called 'up-to techniques' [32]. Among these, one of the most powerful ones is 'up-to expansion and context', whereby the derivatives of two terms can be rewritten using expansion and bisimilarity and then a common context can be erased. Forms of 'bisimulations up-to context' have been shown to be effective

*This is a revised and extended version of a paper presented at the POPL'15 conference, Mumbai, India. 
in various fields, including process calculi [32, 41, 30], $\lambda$-calculi $[22,21,19,42]$, and automata $[8,37]$.

The landmark document for bisimilarity is Milner's CCS book [24]. In the book, Milner carefully explains that the bisimulation proof method is not supposed to be the only method for reasoning about bisimilarity. Indeed, various interesting examples in the book are handled using other techniques, notably unique solution of equations, whereby two tuples of processes are componentwise bisimilar if they are solutions of the same system of equations. (Further techniques exposed in the book include techniques based on axioms and laws, and on modal logic characterisations.) This method is important in verification techniques and tools based on algebraic reasoning [35, 36, 3].

Milner's theorem that guarantees unique solutions [24] has however limitations: the equations must be 'guarded and sequential', that is, the variables of the equations may only be used underneath a visible prefix and preceded, in the syntax tree, only by the sum and prefix operators. This limits the expressiveness of the technique (since occurrences of other operators above the variables, such as parallel composition and restriction, in general cannot be removed), and its transport onto other languages (e.g., languages for distributed systems or higher-order languages, which usually do not include the sum operator).

In this paper we propose a refinement of Milner's technique in which equations are replaced by special inequations called contractions. Intuitively, for a behavioural equivalence $\asymp$, its contraction $\succeq \asymp$ is a preorder in which $P \succeq \asymp Q$ holds if $P \asymp Q$ and, in addition, $Q$ has the possibility of being as efficient as $P$. That is, $Q$ is capable of simulating $P$ by performing less internal work. It is sufficient that $Q$ has one 'efficient' path; $Q$ could also have other paths, that are slower than any path in $P$. Uniqueness of the solution of a system of contractions is defined as with systems of equations: any two solutions must be equivalent with respect to $\asymp$. The difference with equations is in the meaning of solution: in the case of contractions the solution is evaluated with respect to the preorder $\succeq_{\asymp}$, rather than the equivalence $\asymp$.

If a system of equations has a unique solution, then the corresponding system of contractions, obtained by replacing the equation symbol with the contraction symbol, has a unique solution too. The converse however is false: it may be that only the system of contractions has a unique solution. More important, the condition that guarantees a unique solution in Milner's theorem about equations can be relaxed: 'sequentiality' is not required, and 'guardedness' can be replaced by 'weak guardedness', that is, the variables of the contractions can be underneath any prefix, including a prefix representing internal work. (This is the same constraint in Milner's 'unique solution of equations' theorem for strong bisimilarity; the constraint is unsound for equations on weak bisimilarity.)

We show that Milner's theorem is not complete for pure equations (equations in which recursion is only expressible through the variables of the equations, without using the recursion construct of the process language): there are bisimilar processes that cannot be solutions to the same system of guarded and sequential pure equations. In contrast, completeness holds for weakly-guarded pure contractions. The contraction technique is also computationally complete: any bisimulation $\mathcal{R}$ can be transformed into an equivalent system of weaklyguarded contractions that has the same size of $\mathcal{R}$ (where the size of a relation is the number of its pairs, and the size of a system of contractions is the number of its contractions). An analogous result also holds with respect to bisimula- 
tion enhancements such as 'bisimulation up-to expansion and context'. The contraction technique is in fact computationally equivalent to the 'bisimulation up-to contraction and context' technique - a refinement of 'bisimulation up-to expansion and context'.

The contraction technique can be generalised to languages whose syntax is the term algebra derived from some signature, and whose semantics is given as an LTS. In this generalisation the weak-guardedness condition for contractions becomes a requirement of autonomy, essentially saying that the processes that replace the variables of a contraction do not contribute to the initial action of the resulting expression. The technique can also be transported onto other equivalences, including contextually-defined equivalences such as barbed congruence, and non-coinductive equivalences such as contextual equivalence (i.e., may testing) and trace equivalence $[27,11,12]$. For each equivalence, one defines its contraction preorder by controlling the amount of internal work performed.

Finally, we show that a contraction preorder can be injected into the bisimulation game. That is, given an equivalence $\asymp$ and its contraction preorder $\succeq \asymp$, we can define the technique of 'bisimulation up-to $\succeq \asymp$ and context' whereby, in the bisimulation game, the derivatives of the two processes can be manipulated with $\succeq \asymp$ and $\asymp$ (similarly to the manipulations that are possible in the standard 'bisimulation up-to expansion and context' using the expansion relation and bisimilarity) and a common context can then be erased. The resulting 'bisimulation up-to $\succeq \asymp$ and context' is sound for $\asymp$. This technique allows us to derive results for $\asymp$ using the (enhanced) bisimulation proof method.

The contraction technique cannot however be transported onto all (weak) behavioural equivalences. For instance, it does not work in the setting of infinitary trace equivalence (whereby two processes are equal if they have the same finite and infinite traces)[13, 12], and must testing [11]. A discussion on this point is deferred to the concluding section.

We conclude the paper with an example of application of contractions to a higher-order language, which exploits the autonomy condition.

Structure of the paper All background material is reported in Section 2. Contractions and their properties are introduced in Section 3, for bisimilarity and the CCS language. The extension to languages defined from a generic signature is presented in Section 4. The transport of contractions onto other behavioural equivalences is discussed in Sections 5 (barbed congruence), 6 (contextual equivalence), 7 (trace equivalence), and 8 (non-applicability to certain equivalences). The injection of contractions into the bisimulation game is described in Section 9. The example with higher-order languages is reported in Section 10.

\section{Background}

\section{$2.1 \quad$ CCS}

We assume an infinite set of names $a, b, \ldots$ and a set of constant identifiers (or simply constants) for writing recursive processes. The special symbol $\tau$ does not occur in the names and in the constants. The class of the CCS processes is built from the operators of parallel composition, guarded sum, restriction, and 


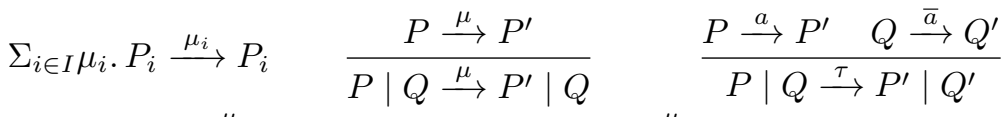

$$
\begin{aligned}
& \frac{P \stackrel{\mu}{\longrightarrow} P^{\prime}}{\boldsymbol{\nu} a P \stackrel{\mu}{\longrightarrow} \boldsymbol{\nu} a P^{\prime}} \mu \neq a, \bar{a} \quad \frac{P \stackrel{\mu}{\longrightarrow} P^{\prime}}{K \stackrel{\mu}{\longrightarrow} P^{\prime}} \text { if } K \triangleq P
\end{aligned}
$$

Figure 1: The LTS for CCS

constants, and the guard of a sum can be an input, an output, or a silent prefix:

$$
\begin{aligned}
P & :=P_{1}\left|P_{2}\right| \Sigma_{i \in I} \mu_{i} . P_{i}|\boldsymbol{\nu} a P| K \\
\mu & :=a|\bar{a}| \tau
\end{aligned}
$$

where $I$ is a countable indexing set. Sums are guarded so to ensure that behavioural equivalences and preorders are substitutive. We write $\mathbf{0}$ when $I$ is empty, and $P+Q$ for binary sums, with the understanding that, to fit the above grammar, $P$ and $Q$ should be sums of prefixed terms. Each constant $K$ that appears in a process should have a process definition, of the form $K \triangleq P$. We sometimes omit trailing $\mathbf{0}$, e.g., writing $a \mid b$ for $a . \mathbf{0} \mid b . \mathbf{0}$. We write $\mu^{n} . P$ for $P$ preceded by $n \mu$-prefixes. In a few examples we write $! \mu$. $P$ as abbreviation for the constant $K_{\mu . P} \triangleq \mu .\left(P \mid K_{\mu . P}\right)$.

The operational semantics is given by means of an LTS, and is reported in Figure 1 (the symmetric version of the two rules for parallel composition has been omitted). The immediate derivatives of a process $P$ are the elements of the set $\left\{P^{\prime} \mid P \stackrel{\mu}{\longrightarrow} P^{\prime}\right.$ for some $\left.\mu\right\}$. We use $\ell$ to range over visible actions (i.e., inputs or outputs, excluding $\tau$ ).

Some standard notations for transitions: $\Longrightarrow$ is the reflexive and transitive closure of $\stackrel{\tau}{\longrightarrow}$, and $\stackrel{\mu}{\Longrightarrow}$ is $\Longrightarrow \stackrel{\mu}{\longrightarrow} \Longrightarrow$ (the composition of the three relations). Moreover, $P \stackrel{\widehat{\mu}}{\longrightarrow} P^{\prime}$ holds if $P \stackrel{\mu}{\longrightarrow} P^{\prime}$ or $\left(\mu=\tau\right.$ and $\left.P=P^{\prime}\right)$; similarly $P \stackrel{\widehat{\mu}}{\longrightarrow} P^{\prime}$ holds if $P \stackrel{\mu}{\Longrightarrow} P^{\prime}$ or $\left(\mu=\tau\right.$ and $\left.P=P^{\prime}\right)$. We write $P(\stackrel{\mu}{\longrightarrow})^{n} P^{\prime}$ if $P$ can become $P^{\prime}$ after performing $n \mu$-transitions. Finally, $P \stackrel{\mu}{\longrightarrow}$ holds if there is $P^{\prime}$ with $P \stackrel{\mu}{\longrightarrow} P^{\prime}$, and similarly for other forms of transitions.

Further notations Letters $\mathcal{R}, \mathcal{S}$ range over relations. We use infix notation for relations, e.g., $P \mathcal{R} Q$ means that $(P, Q) \in \mathcal{R}$. We use a tilde to denote a tuple, with countably many elements; thus the tuple may also be infinite. All notations are extended to tuples componentwise; e.g., $\widetilde{P} \mathcal{R} \widetilde{Q}$ means that $P_{i} \mathcal{R} Q_{i}$, for each component $i$ of the tuples $\widetilde{P}$ and $\widetilde{Q}$. And $C[\widetilde{P}]$ is the process obtained by replacing each hole $[\cdot]_{i}$ of the context $C$ with $P_{i}$. We write $\mathcal{R}^{\mathrm{c}}$ for the closure of a relation under contexts. Thus $P \mathcal{R}^{\mathrm{c}} Q$ means that there are a context $C$ and tuples $\widetilde{P}, \widetilde{Q}$ with $P=C[\widetilde{P}], Q=C[\widetilde{Q}]$ and $\widetilde{P} \mathcal{R} \widetilde{Q}$. We use symbol $\stackrel{\text { def }}{=}$ for abbreviations. For instance, $P \stackrel{\text { def }}{=} G$, where $G$ is some expression, means that $P$ stands for the expression $G$ (in contrast, symbol $\triangleq$ is used for the definition of constants, whereas $=$ is used for syntactic equality and for equations). If $\leq$ is a preorder, then $\geq$ is its inverse (and conversely). 


\subsection{Bisimilarity and expansion}

We focus on weak behavioural equivalences, which abstract from the number of internal steps performed by equivalent processes.

Definition 2.1 (bisimilarity) A process relation $\mathcal{R}$ is a bisimulation if, whenever $P \mathcal{R} Q$, we have:

1. $P \stackrel{\mu}{\longrightarrow} P^{\prime}$ implies that there is $Q^{\prime}$ such that $Q \stackrel{\widehat{\mu}}{\Longrightarrow} Q^{\prime}$ and $P^{\prime} \mathcal{R} Q^{\prime}$;

2. the converse of (1) on the actions from $Q$.

$P$ and $Q$ are bisimilar, written $P \approx Q$, if $P \mathcal{R} Q$ for some bisimulation $\mathcal{R}$.

We sometimes call bisimilarity weak bisimilarity, to distinguish it from strong bisimilarity, $\sim$, obtained by replacing in the above definition the weak answer $Q \stackrel{\widehat{\mu}}{\Longrightarrow} Q^{\prime}$ with the strong $Q \stackrel{\mu}{\longrightarrow} Q^{\prime}$. Other behavioural equivalences, possibly non-coinductive, will be introduced in later sections.

The bisimulation proof method can be enhanced by means of up-to techniques. One of the most useful auxiliary relations in up-to techniques is the expansion relation $\succeq_{\mathrm{e}}[40]$. This is an asymmetric version of $\approx$ where $P \succeq_{\mathrm{e}} Q$ means that $P \approx Q$, but also that $Q$ achieves the same as $P$ with no more work, i.e. with no more $\tau$ actions. Intuitively, if $P \succeq_{\mathrm{e}} Q$, we can think of $Q$ as being at least as fast as $P$ or, more generally, we can think that $P$ uses at least as many resources as $Q$.

Definition 2.2 (expansion) A process relation $\mathcal{R}$ is an expansion if, whenever $P \mathcal{R} Q$

1. $P \stackrel{\mu}{\longrightarrow} P^{\prime}$ implies that there is $Q^{\prime}$ with $Q \stackrel{\widehat{\mu}}{\longrightarrow} Q^{\prime}$ and $P^{\prime} \mathcal{R} Q^{\prime}$;

2. $Q \stackrel{\mu}{\longrightarrow} Q^{\prime}$ implies that there is $P^{\prime}$ with $P \stackrel{\mu}{\Longrightarrow} P^{\prime}$ and $P^{\prime} \mathcal{R} Q^{\prime}$.

$P$ expands $Q$, written $P \succeq_{\mathrm{e}} Q$, if $P \mathcal{R} Q$, for some expansion $\mathcal{R}$.

Relation $\succeq_{\mathrm{e}}$ is studied — using a different terminology — by Arun-Kumar and Hennessy [2]: they show that $\succeq_{\mathrm{e}}$ is a mathematically tractable preorder and has a complete proof system for finite terms based on a modification of the standard $\tau$ laws for CCS. In CCS, strong and weak bisimilarity are congruence relations, and expansion is a precongruence. It holds that $\sim \subseteq_{\mathrm{e}}$ and $\succeq_{\mathrm{e}} \subseteq \approx$; moreover each inclusion is strict. The inclusions are obvious. For the strictness, we have that $P \not \tau . P, P \preceq_{\mathrm{e}} \tau . P$, and $\tau . P \varliminf_{\mathrm{e}} P, \tau . P \approx P$.

A powerful up-to technique is 'bisimulation up-to $\succeq_{\mathrm{e}}$ and context'. It combines 'up-to expansion' (the possibility of rewriting the derivatives of two related processes using $\succeq_{\mathrm{e}}$ and $\approx$ ), with 'up-to context' (the possibility of removing a common context from the derivatives). We recall that $\mathcal{R}^{\mathrm{c}}$ is the context closure of $\mathcal{R}$.

Definition 2.3 (bisimulation up-to $\succeq_{\mathrm{e}}$ and context) A process relation $\mathcal{R}$ is a bisimulation up-to $\succeq_{\mathrm{e}}$ and context if, whenever $P \mathcal{R} Q$, we have:

1. $P \stackrel{\mu}{\longrightarrow} P^{\prime}$ implies that there is $Q^{\prime}$ with $Q \stackrel{\widehat{\mu}}{\Longrightarrow} Q^{\prime}$ and $P^{\prime} \succeq_{\mathrm{e}} \mathcal{R}^{\mathrm{c}} \approx Q^{\prime}$; 
2. the converse of (1) on the actions from $Q$.

The occurrence of $\succeq_{\mathrm{e}}$ on the left of $\mathcal{R}^{\mathrm{c}}$ cannot be replaced by $\approx$, as this would break the soundness of the technique [32]. The technique is sound [38]:

Lemma 2.4 (soundness of bisimulation up-to $\succeq_{\mathrm{e}}$ and context) If $\mathcal{R}$ is a bisimulation up-to $\succeq_{\mathrm{e}}$ and context, then $R \subseteq \approx$.

\subsection{An example}

In examples in the paper, we sometimes use a version of CCS with value passing; this could be translated into pure CCS [24], but having explicit value passing improves readability. In a value-passing calculus, $a(x) . P$ is an input at $a$ in which $x$ is the placeholder for the value received, whereas $\bar{a}\langle n\rangle . P$ is an output at $a$ of the value $n$; and $A\langle n\rangle$ is a parametrised constant. The following example illustrates 'bisimulation up-to $\succeq_{\mathrm{e}}$ and context', and will then be used for comparison with other techniques.

We wish to implement a server that, when interrogated by clients at a channel $c$, starts a certain interaction protocol with the client, after consulting an auxiliary server $A$ at $a$. Here the auxiliary server $A$ is deterministic: at every cycle it outputs an integer value, which changes with the cycle (this change is represented by the successor function, for simplicity).

We consider two implementations of the server. The difference between them is that the first server, $L$, is 'lazy', and consults $A$ only after a request from a client has been received. In contrast, the other server, $E$, is 'eager', and consults $A$ beforehand, so then to be ready in answering a client:

$$
\begin{aligned}
L & \triangleq c(z) \cdot a(x) \cdot(L \mid R\langle c, x, z\rangle) \\
E & \triangleq a(x) \cdot c(z) \cdot(E \mid R\langle c, x, z\rangle) \\
A\langle n\rangle & \triangleq \bar{a}\langle n\rangle \cdot A\langle n+1\rangle
\end{aligned}
$$

Here $R\langle c, x, z\rangle$ represents the interaction protocol that is started with a client, and can be any process. It may use the values $x$ and $z$ (obtained from the client and the auxiliary server $A$ ); the interactions produced may indeed depend on the values $x$ and $z$. Process $R\langle c, x, z\rangle$ may also use channel $c$, and therefore trigger further interactions with the server; in contrast, $R\langle c, x, z\rangle$ may not use $a$ (i.e., it may not interrogate the auxiliary server).

We use the 'bisimulation up-to expansion and context' technique to prove that the composition of the two servers with $A$ yields bisimilar lazy and eager systems:

$$
\begin{array}{lll}
L S\langle n\rangle & \stackrel{\text { def }}{=} & \boldsymbol{\nu} a(A\langle n\rangle \mid L) \\
E S\langle n\rangle & \stackrel{\text { def }}{=} & \boldsymbol{\nu} a(A\langle n\rangle \mid E)
\end{array}
$$

Relation $\mathcal{R} \stackrel{\text { def }}{=} \cup_{n}\{(L S\langle n\rangle, E S\langle n\rangle)\}$ is a bisimulation up-to expansion and context. Consider a pair $(L S\langle n\rangle, E S\langle n\rangle)$. The two processes have one initial transition; the most interesting case is the challenge transition from $E S\langle n\rangle$, and we only consider this one. We have

$$
E S\langle n\rangle \stackrel{\tau}{\longrightarrow} \boldsymbol{\nu} a(A\langle n+1\rangle \mid c(z) .(E \mid R\langle c, n, z\rangle)) \stackrel{\text { def }}{=} E^{\prime}
$$


Process $L S\langle n\rangle$ may not produce internal steps, hence its only possible answer is

$$
L S\langle n\rangle \Longrightarrow L S\langle n\rangle
$$

We can now perform some algebraic manipulations of $E^{\prime}$ : first, we employ the CCS expansion law to pull out the prefix at $c$, then a structural law to resize the scope of the restriction at $a$ in which we exploit the property that $R\langle c, n, z\rangle$ may not use $a$. (All these laws are valid for strong bisimilarity, hence also for expansion.) We thus obtain:

$$
\begin{array}{rl}
E^{\prime} \quad \succeq_{\mathrm{e}} & c(z) \cdot(\boldsymbol{\nu} a(A\langle n+1\rangle \mid E) \mid R\langle c, n, z\rangle) \\
& =c(z) \cdot(E S\langle n+1\rangle \mid R\langle c, n, z\rangle) \stackrel{\text { def }}{=} E^{\prime \prime}
\end{array}
$$

We can act similarly on $L S\langle n\rangle$, and in addition also employing the law

$$
\boldsymbol{\nu} a(a(y) \cdot P \mid \bar{a}\langle v\rangle \cdot Q) \approx \boldsymbol{\nu} a(P\{v / y\} \mid Q)
$$

This gives us:

$$
\begin{aligned}
L S\langle n\rangle & \approx c(z) \cdot(\boldsymbol{\nu} a(A\langle n+1\rangle \mid L) \mid R\langle c, n, z\rangle) \\
& =c(z) \cdot(L S\langle n+1\rangle \mid R\langle c, n, z\rangle) \stackrel{\text { def }}{=} L^{\prime}
\end{aligned}
$$

We have thus obtained two processes, $E^{\prime \prime}$ and $L^{\prime}$, in the context closure of $\mathcal{R}$, and we are done.

In the proof, the 'up-to' techniques allow us to work with a relation that has exactly one pair for each integer. Specifically, 'up-to context' avoids us considering processes in parallel with the lazy and eager systems, whereas 'upto expansion' allows us to reason only on the 'normal forms' $L S\langle n\rangle$ and $E S\langle n\rangle$ for these systems (avoiding us to take all their reachable states into account).

\subsection{Systems of equations}

Uniqueness of solutions of equations [24] intuitively says that if a context $C$ obeys certain conditions, then all processes $P$ that satisfy the equation $P \approx C[P]$ are bisimilar with each other.

We need variables to write equations. We use capital letters $X, Y, Z$ for these variables and call them equation variables. The body of an equation is a CCS expression possibly containing equation variables. Thus such expressions, ranged over by $E$, live in the CCS grammar extended with equation variables.

Definition 2.5 Assume that, for each $i$ of a countable indexing set $I$, we have variables $X_{i}$, and expressions $E_{i}$ possibly containing such variables. Then

$$
\left\{X_{i}=E_{i}\right\}_{i \in I}
$$

is a system of equations. (There is one equation for each variable $X_{i}$.)

We write $E[\widetilde{P}]$ for the expression resulting from $E$ by replacing each variable $X_{i}$ with the process $P_{i}$, assuming $\widetilde{P}$ and $\widetilde{X}$ have the same length. (This is syntactic replacement, akin to the substitution of the holes of a context with processes.) The components of $\widetilde{P}$ need not be different from each other, as it must be for the variables $\widetilde{X}$. If the system has infinitely many equations, the tuples $\widetilde{P}$ and $\widetilde{X}$ are infinite too. 
Definition 2.6 Suppose $\left\{X_{i}=E_{i}\right\}_{i \in I}$ is a system of equations:

- $\widetilde{P}$ is a solution of the system of equations for $\approx$ if for each $i$ it holds that $P_{i} \approx E_{i}[\widetilde{P}]$.

- the system has a unique solution for $\approx$ if whenever $\widetilde{P}$ and $\widetilde{Q}$ are both solutions for $\approx$, then $\widetilde{P} \approx \widetilde{Q}$.

Examples of systems with a unique solution for $\approx$ are:

1. $X=a . X$

2. $X_{1}=a \cdot X_{2}, X_{2}=b \cdot X_{1}$

The unique solution of the system (1), modulo $\approx$, is the constant $K \triangleq a$. $K$ : for any other solution $P$ we have $P \approx K$. The unique solution of (2), modulo $\approx$, are the constants $K_{1}, K_{2}$ with $K_{1} \triangleq a . K_{2}$ and $K_{2} \triangleq b . K_{1}$; again, for any other pair of solutions $P_{1}, P_{2}$ we have $K_{1} \approx P_{1}$ and $K_{2} \approx P_{2}$. Examples of systems that do not have a unique solution are:

1. $X=X$

2. $X=\tau \cdot X$

3. $X=a \mid X$

All processes are solutions of (1) and (2); examples of solutions for (3) are $K$ and $K \mid b$, for $K \triangleq a . K$.

Definition 2.7 A system of equations $\left\{X_{i}=E_{i}\right\}_{i \in I}$ is

- guarded if, in each $E_{i}$, each occurrence of an equation variable is underneath a visible prefix;

- sequential if, in each $E_{i}$, each occurrence of an equation variable only appears underneath prefixes and sums.

In other words, if the system is sequential, then for every expression $E_{i}$, any subexpression of $E_{i}$ in which $X_{j}$ appears, apart from $X_{j}$ itself, is a sum (of prefixed terms). For instance,

- $X=\tau . X+\mu .0$ is sequential but not guarded, because the guarding prefix for the variable is not visible.

- $X=\ell . X \mid P$ is guarded but not sequential.

- $X=\ell . X+\tau \cdot \boldsymbol{\nu} a(a . \bar{b} \mid a . \mathbf{0})$, as well as $X=\tau \cdot(a . X+\tau . b . X+\tau)$ are both guarded and sequential.

Theorem 2.8 (unique solution of equations, [24]) A system of guarded and sequential equations has a unique solution for $\approx$. 
The proof exploits an invariance property on immediate transitions for guarded and sequential expressions, and then extracts a bisimulation (up-to bisimilarity) out of the solutions of the system. To see the need of the sequentiality condition, consider the equation (from [24])

$$
X=\boldsymbol{\nu} a(a . X \mid \bar{a})
$$

where $X$ is guarded but not sequential. Any processes that does not use $a$ is a solution.

\section{Contractions}

In Theorem 2.8 the constraints on guardedness and, especially, on sequentiality limit its applicability. Essentially, it can only be applied when the only process operators in the equations are prefixing and sum. Further, the same definitions and examples discussed for bisimilarity (and hence also the same limitations) apply to other behavioural equivalences; e.g., contextual equivalence and tracebased equivalences.

One may wonder if the conditions of Theorem 2.8 can be relaxed by simply requiring that each equation be sequentially guarded, that is, of the form $X=$ $\Sigma_{j} \ell_{j} . E_{j}$ (where $\ell_{j}$ is a visible action). Unfortunately, uniqueness still fails; a counterexample is

$$
X=a . \nu a(\bar{a} \mid X) .
$$

Any process $P$ with $P \approx a . P^{\prime}$, and $P^{\prime}$ unable to use $a$, (i.e., $a$ is not in the sort of $P^{\prime}$ ), is a solution. Examples are $a . \mathbf{0}$ and $a . b . \mathbf{0}$.

An equation $X=a$. $E$ need not have a unique solution even if we confine ourselves to processes that may only perform $a$ transitions. An example is the equation

$$
X=a . \nu b(\boldsymbol{\nu} a(\bar{a} \cdot ! \bar{a} \cdot \bar{b} \mid X) \mid ! b . a) .
$$

Here the body of the equation produces an $a$, cancels the first $a$ from $X$ and then reproduces all other $a$ 's. Any process $P$ with $P \approx a . P^{\prime}$ for some $P^{\prime}$, is a solution; for instance, $a . \mathbf{0}$ or $a . a . \mathbf{0}$ or even !a. $\mathbf{0 .}$

Remark 3.1 The unique-solution method incorporates the flavour of 'up-to context': for equations $\widetilde{X}=\widetilde{E}$, finding solutions $\widetilde{P}$ and $\widetilde{Q}$ means showing that the behaviours of corresponding elements $P_{i}$ and $Q_{i}$ of the solution can be given a structure, represented by $E_{i}$, which may make use of other elements of the solution (represented by the occurrences of the variables in $E_{i}$ ). However, the sequentiality condition makes the up-to context useless: when $X$ in $E$ is reached, there is no 'context' left.

\subsection{Contraction preorders}

The constraints on the unique-solution Theorem 2.8 can be weakened if we move from equations to certain inequations that we call contractions.

Intuitively, for a behavioural equivalence $\asymp$, its contraction $\succeq \asymp$ is a preorder in which $P \succeq \asymp Q$ holds if $P \asymp Q$ and, in addition, $Q$ has the possibility of being at least as efficient as $P$. That is, if $P$ can do some work (i.e., some interactions with its environment), then $Q$ should be able to do the same work 
at least as quickly as $P$ (i.e., performing no more $\tau$-steps then those performed by $P$ ). Process $Q$, however, may be nondeterministic and may have other ways of doing the same work, and these could be slow (i.e., involving more $\tau$-steps than those performed by $P$ ). Thus we cannot really say that ' $Q$ is more efficient than $P^{\prime}$, as we could have done if we had followed the schema of the expansion preorder of Section 2.2 .

We explain the idea of contraction on the concrete case of weak bisimilarity, and then generalise it.

Definition 3.2 (bisimulation contraction) A process relation $\mathcal{R}$ is a bisimulation contraction if, whenever $P \mathcal{R} Q$,

1. $P \stackrel{\mu}{\longrightarrow} P^{\prime}$ implies there is $Q^{\prime}$ such that $Q \stackrel{\widehat{\mu}}{\longrightarrow} Q^{\prime}$ and $P^{\prime} \mathcal{R} Q^{\prime}$;

2. $Q \stackrel{\mu}{\longrightarrow} Q^{\prime}$ implies there is $P^{\prime}$ such that $P \stackrel{\widehat{\mu}}{\Longrightarrow} P^{\prime}$ and $P^{\prime} \approx Q^{\prime}$.

Bisimilarity contraction, written $\succeq_{\text {bis }}$, is the union of all bisimulation contractions.

In the first clause $Q$ is required to match $P$ 's challenge transition with at most one transition. This makes sure that $Q$ is capable of mimicking $P$ 's work at least as efficiently as $P$. In contrast, the second clause of Definition 3.2, on the challenges from $Q$, entirely ignores efficiency: it is the same clause of weak bisimulation - the final derivatives are even required to be related by $\approx$, rather than by $\mathcal{R}$.

Bisimilarity contraction is coarser than the expansion relation $\succeq_{\text {e }}$ of Definition 2.2. Clause (1) is the same in the two definitions. But in clause (2) expansion uses $P \stackrel{\mu}{\Longrightarrow} P^{\prime}$, rather than $P \stackrel{\widehat{\mu}}{\Longrightarrow} P^{\prime}$; moreover with contraction the final derivatives are simply required to be bisimilar. An expansion $P \succeq_{\mathrm{e}} Q$ tells us that $Q$ is always at least as efficient as $P$, whereas the contraction $P \succeq_{\text {bis }} Q$ just says that $Q$ has the possibility of being at least as efficient as $P$.

Example 3.3 We have $a \succeq_{\text {bis }} \tau$. $a$. However, $a+\tau$. $a \succeq_{\text {bis }} a$, as well as its converse, $a \succeq_{\text {bis }} a+\tau$. $a$. Indeed, if $P \approx Q$ then $P \succeq_{\text {bis }} P+Q$. The last two relations do not hold with $\succeq_{\mathrm{e}}$, which explains the strictness of the inclusion $\succeq_{\mathrm{e}} \subseteq \succeq_{\text {bis. }}$

As bisimilarity contraction follows expansion in one direction and bisimilarity in the other, clearly separating the two, the precongruence and congruence for such relations can be combined into a precongruence proof for the contraction.

Theorem 3.4 $\succeq_{\text {bis }}$ is a precongruence in CCS.

Proof The proof is similar to analogous proofs for bisimilarity and expansion. As an example, to show that $\succeq_{\text {bis }}$ is preserved by parallel composition one shows that the relation

$$
\left\{(P|R, Q| R) \mid P \succeq_{\text {bis }} Q\right\}
$$

is a bisimulation contraction. When analising the challenges from $P$, one uses clause (1) of Definition 3.2, which is the same clause in the definition of expansion, and reasons as in the analogous proof of precongruence for expansion. Thus 
if $P\left|R \stackrel{\mu}{\longrightarrow} P^{\prime}\right| R^{\prime}$ one distinguishes the cases when $P$ alone moves, $R$ alone moves, and both $P$ and $R$ move. We only consider the third case, the others being simpler. Thus suppose that $\mu=\tau, P \stackrel{a}{\rightarrow} P^{\prime}$, and $R \stackrel{\bar{a}}{\rightarrow} R^{\prime}$. From $P \succeq_{\text {bis }} Q$ we deduce $Q \stackrel{a}{\rightarrow} Q^{\prime}$ with $P^{\prime} \succeq_{\text {bis }} Q^{\prime}$, and therefore also $Q\left|R \stackrel{\tau}{\rightarrow} Q^{\prime}\right| \bar{R}^{\prime}$ with $P^{\prime}\left|R^{\prime} \mathcal{R} Q^{\prime}\right| R^{\prime}$

When the challenges are from $Q$, in contrast, one reasons using clause (2) of Definition 3.2, which is the same clause of the definition of weak bisimulation, and therefore one can follow the reasoning in the congruence proof of weak bisimulation.

\subsection{Systems of contractions}

A system of contractions is defined as a system of equations, except that the contraction symbol $\succeq$ is used in the place of the equality symbol $=$. Thus a system of contractions is a set $\left\{X_{i} \succeq E_{i}\right\}_{i \in I}$ where $I$ is an indexing set and expressions $E_{i}$ may contain the contraction variables $\left\{X_{i}\right\}_{i \in I}$.

Definition 3.5 Given a behavioural equivalence $\asymp$ and its contraction $\succeq \asymp$, and a system of contractions $\left\{X_{i} \succeq E_{i}\right\}_{i \in I}$, we say that:

- $\widetilde{P}$ is a solution for $\succeq \asymp$ of the system of contractions if $\widetilde{P} \succeq \asymp \widetilde{E}[\widetilde{P}]$;

- the system has a unique solution for $\asymp$ if whenever $\widetilde{P}$ and $\widetilde{Q}$ are both solutions for $\succeq \asymp$ then $\widetilde{P} \asymp \widetilde{Q}$.

When we reason about bisimilarity, the contraction symbol $\succeq$ is interpreted as the bisimilarity contraction $\succeq_{\text {bis }}$, and the equivalence $\asymp$ as the bisimilarity $\approx$. Thus $\widetilde{P}$ being a solution for $\succeq_{\text {bis }}$ of the system of contractions $\left\{X_{i} \succeq E_{i}\right\}_{i \in I}$ means that $\widetilde{P} \succeq_{\text {bis }} \widetilde{E}[\widetilde{P}]$; and the system having a unique solution for $\approx$ means that whenever $\widetilde{P}$ and $\widetilde{Q}$ are both solutions for $\succeq_{\text {bis }}$ then $\widetilde{P} \approx \widetilde{Q}$.

Lemma 3.6 If a system of equations $\left\{X_{i}=E_{i}\right\}_{i \in I}$ has a unique solution for $\approx$, then also the corresponding system of contractions $\left\{X_{i} \succeq E_{i}\right\}_{i \in I}$ has a unique solution for $\approx$.

Proof Any solution for $\succeq \asymp$ of the system of contractions is a solution for $\approx$ of the corresponding system of equations. Hence if the latter system has a unique solution for $\approx$ then also the former has a unique solution $\approx$.

The converse of the lemma, in contrast, is false: as we shall see, systems of contractions more easily have a unique solution.

Remark 3.7 Any system of equations or contractions has at least one solution for strong bisimilarity, obtained by interpreting the equations as recursive process definitions. That is, for equations one associates to each equation $X_{i}=E_{i}$ a fresh constant $K_{i}$ with definition $K_{i} \triangleq E\{\widetilde{K} / \widetilde{X}\}$. Then $\widetilde{K}$ is a solution to the system of equations for strong bisimilarity, hence also for weak bisimilarity; and it is also a solution for $\succeq \asymp$ of the corresponding system of contractions $\left\{X_{i} \succeq E_{i}\right\}_{i \in I}$. (We are assuming here that the alphabet of constants always containts enough 'fresh' constants; for instance one may assume that it is an uncountable set.) 
We now study conditions that guarantee unique solutions for $\approx$ of systems of contractions.

\subsection{Conditions for unique solution of contractions}

For contraction, the following weak-guardedness condition is sufficient to have a unique solution. The condition is weaker than the guardedness condition because the guarding prefix can be any prefix, not necessarily a visible one.

Definition 3.8 A system of contractions $\left\{X_{i} \succeq E_{i}\right\}_{i \in I}$ is weakly guarded if, in each $E_{i}$, each occurrence of a contraction variable is underneath a prefix.

In proofs about weakly-guarded contractions we will often unfold the contractions, exploiting the substitutivity of the contraction preorder, with the objective of placing processes that are solutions of the contractions underneath a certain number of prefixes. Suppose $\widetilde{P}$ are solutions of a system of contractions $\left\{X_{i} \succeq E_{i}\right\}_{i \in I}$, and consider a context $C[\widetilde{P}]$. Then the process obtained from $C[\widetilde{P}]$ by unfolding the contractions once is $C[\widetilde{E}[\widetilde{P}]]$; the process obtained by unfolding the contractions twice is $C[\widetilde{E}[\widetilde{E}[\widetilde{P}]]]$; and similarly for the $n$-unfolding.

Lemma 3.9 Suppose $\widetilde{P}$ and $\widetilde{Q}$ are solutions for $\approx$ of a system of weaklyguarded contractions. For any context $C$, if $C[\widetilde{P}] \stackrel{\mu}{\Longrightarrow} R$, then there is a context $C^{\prime}$ such that $R \succeq_{\text {bis }} C^{\prime}[\widetilde{P}]$ and $C[\widetilde{Q}] \stackrel{\widehat{\mu}}{\Longrightarrow} \approx C^{\prime}[\widetilde{Q}]$.

Proof Let $n$ be the length of the transition $C[\widetilde{P}] \stackrel{\mu}{\Longrightarrow} R$ (the number of 'strong steps' of which it is composed), and let $C^{\prime \prime}[\widetilde{P}]$ and $C^{\prime \prime}[\widetilde{Q}]$ be the processes obtained from $C[\widetilde{P}]$ and $C[\widetilde{Q}]$ by unfolding the definitions of the contractions $n$ times. Thus in $C^{\prime \prime}$ each hole is underneath at least $n$ prefixes, and cannot contribute to an action in the first $n$ transitions.

Since both $\widetilde{P}$ and $\widetilde{Q}$ are solutions of the system of contractions, by the precongruence properties of $\succeq_{\text {bis }}$ we have $C[\widetilde{P}] \succeq_{\text {bis }} C^{\prime \prime}[\widetilde{P}]$ and $C[\widetilde{Q}] \succeq_{\text {bis }} C^{\prime \prime}[\widetilde{Q}]$. Moreover, since each hole of the context $C^{\prime \prime}$ is underneath at least $n$ prefixes, applying the definition of $\succeq_{\text {bis }}$ on the transition $C[\widetilde{P}] \stackrel{\mu}{\Longrightarrow} R$ and reasoning by induction on $n$, we infer the existence of $C^{\prime}$ such that

$$
C^{\prime \prime}[\widetilde{P}] \stackrel{\widehat{\mu}}{\Longrightarrow} C^{\prime}[\widetilde{P}] \preceq_{\text {bis }} R
$$

and

$$
C^{\prime \prime}[\widetilde{Q}] \stackrel{\widehat{\mu}}{\Longrightarrow} C^{\prime}[\widetilde{Q}]
$$

Finally, again applying the definition of $\succeq_{\text {bis }}$ on $C[\widetilde{Q}] \succeq_{\text {bis }} C^{\prime \prime}[\widetilde{Q}]$, we derive

$$
C[\widetilde{Q}] \stackrel{\widehat{\mu}}{\Longrightarrow} \approx C^{\prime}[\widetilde{Q}]
$$

Theorem 3.10 (unique solution of contractions for $\approx$ ) A system of weaklyguarded contractions has a unique solution for $\approx$. 
Proof Suppose $\widetilde{P}$ and $\widetilde{Q}$ are solutions of a system of weakly-guarded contractions, and consider the relation

$$
\mathcal{R} \stackrel{\text { def }}{=}\{(R, S) \mid R \approx C[\widetilde{P}], S \approx C[\widetilde{Q}] \text { for some context } C\} .
$$

We show that $\mathcal{R}$ is a bisimulation. Suppose $R \mathcal{R} S$ via the context $C$, and $R \stackrel{\mu}{\longrightarrow} R^{\prime}$. We have to find $S^{\prime}$ with $S \stackrel{\widehat{\mu}}{\longrightarrow} S^{\prime}$ and $R^{\prime} \mathcal{R} S^{\prime}$. From $R \approx C[\widetilde{P}]$, we derive $C[\widetilde{P}] \stackrel{\widehat{\mu}}{\Longrightarrow} R^{\prime \prime} \approx R^{\prime}$, for some $R^{\prime \prime}$. By Lemma 3.9 , there is $C^{\prime}$ with $R^{\prime \prime} \succeq_{\text {bis }} C^{\prime}[\widetilde{P}]$ and $C[\widetilde{Q}] \stackrel{\widehat{\mu}}{\Longrightarrow} \approx C^{\prime}[\widetilde{Q}]$. Hence by definition of $\approx$, there is also $S^{\prime}$ with $S \stackrel{\widehat{\mu}}{\Longrightarrow} S^{\prime} \approx C^{\prime}[\widetilde{Q}]$. This closes the proof, as we have $R^{\prime} \approx C^{\prime}[\widetilde{P}]$ and $S^{\prime} \approx C^{\prime}[\widetilde{Q}]$.

In comparison to Theorem 2.8 for equations, in Theorem 3.10 for contractions the 'guardedness' condition is weakened, allowing variables that are underneath $\tau$ prefixes; most important, the sequentiality condition is removed, allowing variables underneath any process constructs.

Example 3.11 The following contractions have a unique solution for $\approx$ :

1. $X \succeq \tau . X$

2. $X \succeq a . \nu a(\bar{a} \mid X))$

3. $X \succeq a . \nu b(\boldsymbol{\nu} a(\bar{a} . ! a . \bar{b} \mid X) \mid ! b . a)$

We have seen in Section 2.4 and at the beginning of Section 3 that the corresponding equations do not have a unique solution. The solutions of the contraction (1) are all inactive processes, where a process is inactive if it cannot perform visible actions (i.e., if $P$ is the process, then there is no $P^{\prime}$ and visible action $\ell$ such that $P \Longrightarrow P^{\prime} \stackrel{\ell}{\rightarrow}$ ). The contraction has a unique solution because all inactive processes are bisimilar. It is easy to see that an inactive process is solution. Conversely, suppose $P$ is not inactive, and let $n$ be the least $n \geq 0$ such that $P(\stackrel{\tau}{\longrightarrow})^{n} \stackrel{\ell}{\rightarrow}$ for some $\ell$; then $P$ is not a solution of $P \succeq \tau$. $P$ because $\tau$. $P$ needs at least $n+1 \tau$-steps before exhibiting any visible action, and therefore can never be more efficient than $P$. Example of solutions for (2) and (3) are a.P and $\tau$.a.P, where $P$ is inactive. Any solution of (2) and (3) is bisimilar with $a .0$.

Remark 3.12 Results such as Lemma 3.9 and Theorem 3.10 also hold if the game played in clause (1) of Definition 3.2 of bisimulation contraction is that of strong simulation (i.e., " $P \stackrel{\mu}{\longrightarrow} P^{\prime}$ implies there is $Q^{\prime}$ such that $Q \stackrel{\mu}{\longrightarrow} Q^{\prime}$ and $\left.P^{\prime} \mathcal{R} Q^{\prime \prime \prime}\right)$. However, the resulting relation would not be coarse enough to capture expansion - a major goal for this paper is understanding existing 'bisimilarity up-to' techniques, where expansion is important.

\subsection{Completeness}

An interesting class of contractions are those in which the body $E$ of each contraction $X \succeq E$ does not contain constants. In these systems, all forms of infinity in the behaviour of processes are captured by recursive calls through 
the contraction variables. We call such systems pure. Similarly we call pure a system of equations without constants. Pure contractions and equations are the kind of contractions or equations that we would normally write when reasoning on systems. In this section we discuss the expressiveness of pure systems of contractions and equations. (With constants the question is vacuous, as the behaviour of any process $P$ is captured by the guarded and sequential equation $X=P$.) We show that the technique of weakly-guarded contractions given by Theorem 3.10 is complete, whereas that of guarded and sequential equations given by Theorem 2.8 is not.

If $\mathcal{R}$ is a relation then we can also view $\mathcal{R}$ as an ordered sequence of pairs (e.g., assuming some lexicographical ordering). Then $\mathcal{R}_{i}$ indicates the tuple obtained by projecting the pairs in $\mathcal{R}$ on the $i$-th component $(i=1,2)$.

Theorem 3.13 (completeness) Suppose $\mathcal{R}$ is a bisimulation. Then there is a system of weakly-guarded pure contractions of which $\mathcal{R}_{1}$ and $\mathcal{R}_{2}$ are solutions for $\succeq_{\text {bis. }}$.

Proof Suppose $\mathcal{R}$ is a bisimulation. We define a system of contractions of which $\mathcal{R}_{1}$ and $\mathcal{R}_{2}$ are solutions. The variables of the contractions are of the form $X_{P, Q}$ for $P \mathcal{R} Q$, and there is one contraction for each pair in $\mathcal{R}$.

We show how the contraction for a pair $P \mathcal{R} Q$ is built. Consider an enumeration of all the transitions from $P$ :

$$
P \stackrel{\mu_{r}}{\longrightarrow} P_{r}
$$

where $r$ ranges over some countable set $I_{P}$. Following the bisimulation game, for each $r$ there is $Q_{r}$ s.t. $Q \stackrel{\widehat{\mu_{r}}}{\Longrightarrow} Q_{r}$ and $P_{r} \mathcal{R} Q_{r}$. Proceeding similarly on the challenge transitions from $Q$, i.e. $Q \stackrel{\mu_{s}}{\longrightarrow} Q_{s}$ for $s \in I_{Q}$, we find processes $P_{s}$ with $P \stackrel{\widehat{\mu_{s}}}{\Longrightarrow} P_{s}$ and $P_{s} \mathcal{R} Q_{s}$. Then the contraction for the pair $P, Q$ is:

$$
X_{P, Q} \succeq \Sigma_{r} \mu_{r} . X_{P_{r}, Q_{r}}+\Sigma_{s} \mu_{s} . X_{P_{s}, Q_{s}}
$$

The resulting system of contractions is weakly guarded. We now show that $\mathcal{R}_{1}$ and $\mathcal{R}_{2}$ are solutions.

Consider an equation (2), in which the transitions from $P$ and $Q$ are enumerated using the indexes $r$ and $s$ as above. We show that the challenge transitions from $P$ are matched by $\Sigma_{r} \mu_{r} . P_{r}+\Sigma_{s} \mu_{s} . P_{s}$ as by clause (1) of Definition 3.2, and the converse using clause (2). (One proceeds similarly for $Q$ in place of $P$.)

If the challenge is $P \stackrel{\mu_{r}}{\longrightarrow} P_{r}$, the answer can simply be

$$
\Sigma_{r} \mu_{r} . P_{r}+\Sigma_{s} \mu_{s} . P_{s} \stackrel{\mu_{r}}{\longrightarrow} P_{r}
$$

since $\succeq_{\text {bis }}$ is reflexive. For the converse, the interesting case is a challenge of the form

$$
\Sigma_{r} \mu_{r} . P_{r}+\Sigma_{s} \mu_{s} . P_{s} \stackrel{\mu_{s}}{\longrightarrow} P_{s}
$$

In this case the answer is $P \stackrel{\widehat{\mu_{s}}}{\Longrightarrow} P_{s}$.

The contractions in the proof of the theorem are sequential and weakly guarded, but not necessarily guarded. 
Remark 3.14 In the final step of the proof above, relation $\stackrel{\widehat{\mu_{s}}}{\Longrightarrow}$ comes from the definition of weak bisimulation, and could not be replaced by $\stackrel{\mu_{s}}{\Longrightarrow}$. This explains why the completeness proof fails with expansion in place of contraction.

The assertion of Theorem 3.13 can actually be refined: the technique based on weakly-guarded contractions is also computationally complete with respect to the bisimulation proof method, in the sense that the size of the structures needed and the subsequent amount of checks are comparable. The size of a relation is the number of its pairs. The size of a system of contractions is the number of contractions. The proof of Theorem 3.13 shows that the system of contractions derived from a bisimulation $\mathcal{R}$ has the same size as $\mathcal{R}$; moreover, the work needed to prove that $\mathcal{R}_{1}$ and $\mathcal{R}_{2}$ are solutions of the system of contractions is precisely the work needed to check the challenge/response diagrams of the bisimulation game for $\mathcal{R}$.

In contrast, the method for equations resulting from Theorem 2.8 is not complete. For instance, there is no system of guarded and sequential pure equations in which one of the solutions is the process $K$ so defined:

$$
K \triangleq \tau .(a \mid K)+\tau . \mathbf{0} .
$$

To see this, it is useful to express the behaviour of $K$ via the following constants:

$$
\begin{aligned}
H_{0} & \triangleq \tau \cdot H_{1}+\tau \\
H_{i} & \triangleq \tau \cdot H_{i+1}+a \cdot H_{i-1}+\tau \cdot a^{i} \quad(i>0)
\end{aligned}
$$

We have $a^{i} \mid K \approx H_{i}$, for each $i$, as witnessed by the relation

$$
\mathcal{R} \stackrel{\text { def }}{=} \cup_{i \geq 0}\left\{\left(a^{i} \mid K, H_{i}\right)\right\},
$$

which is a bisimulation up-to strong bisimilarity. Now, for each $n \neq m$, we have $H_{n} \not \approx H_{m}$ (because, assuming $n<m, H_{m}$ cannot match the transition $\left.H_{n} \stackrel{\tau}{\longrightarrow} a^{n}\right)$; moreover, for each $n$ there is a transition $H_{n} \stackrel{\tau}{\longrightarrow} H_{n+1}$. As a consequence, the infinite sequence of transitions

$$
H_{0} \stackrel{\tau}{\rightarrow} H_{1} \stackrel{\tau}{\longrightarrow} \cdots H_{n} \stackrel{\tau}{\rightarrow} H_{n+1} \stackrel{\tau}{\longrightarrow} \cdots
$$

goes through states that are pairwise non-bisimilar. An equation of which $K$ is solution should be able to express the same behaviour. This is impossible, however, if the equation is sequential and guarded, because the equation variables must be underneath a visible prefix, and can only be reached by performing a visible action. Hence an infinite nesting of internal transitions as in (3) cannot be derived.

\subsection{Relationship with up-to context}

The completeness of the contraction technique given by Theorem 3.13, including the computational completeness discussed after the theorem, remains also with respect to powerful enhancements of the bisimulation proof method such as 'up-to context' techniques.

We show that the contraction technique is in fact computationally equivalent to the 'up-to $\succeq_{\text {bis }}$ and context' technique, a refinement of the 'up-to expansion and context' of Definition 2.3 (the former captures a larger set of relations because bisimilarity contraction is coarser than expansion). 
Definition 3.15 (bisimulation up-to $\succeq_{\text {bis }}$ and context) A process relation $\mathcal{R}$ is a bisimulation up-to $\succeq_{\text {bis }}$ and context if, whenever $P \mathcal{R} Q$, we have:

1. $P \stackrel{\mu}{\longrightarrow} P^{\prime}$ implies there is $Q^{\prime}$ such that $Q \stackrel{\widehat{\mu}}{\Longrightarrow} Q^{\prime}$ and $P^{\prime} \succeq_{\text {bis }} \mathcal{R}^{\mathrm{c}} \approx Q^{\prime}$

2. the converse of (1) on the actions from $Q$.

Theorem 3.16 Suppose $\mathcal{R}$ is a bisimulation up-to $\succeq_{\text {bis }}$ and context. Then there is a system of weakly-guarded contractions, of the same size as $\mathcal{R}$, of which $\mathcal{R}_{1}$ and $\mathcal{R}_{2}$ are solutions for $\succeq_{\text {bis }}$.

Conversely, suppose $\widetilde{P}$ and $\widetilde{Q}$ are solutions for $\succeq_{\text {bis }}$ to the same system of weakly-guarded contractions. Then the relation $\left\{\left(P_{i}, Q_{i}\right)\right\}_{i}$ is a bisimulation up-to $\succeq_{\text {bis }}$ and context.

Proof The first part of the theorem is proved along the lines of the proof of Theorem 3.13 (we are in fact strengthening Theorem 3.13); we have however to take contexts into account, thus the contraction variables may end up within an arbitrary context. Specifically, suppose $\mathcal{R}$ is a bisimulation up-to $\succeq_{\text {bis }}$ and context. We define the contractions satisfied by $\mathcal{R}_{1}$ and $\mathcal{R}_{2}$. Each pair in $\mathcal{R}$ gives rise to a contraction. We use the contraction variable $X_{i}$ for the contraction generated by the $i$-th pair in $\mathcal{R}$. In the solutions of the contractions, $X_{i}$ is replaced by the $i$-th process in $\mathcal{R}_{1}$ or $\mathcal{R}_{2}$.

Consider $P \mathcal{R} Q$, and an enumeration of all the transitions from $P$ and $Q$

$$
P \stackrel{\mu_{r}}{\longrightarrow} P_{r}
$$

and

$$
Q \stackrel{\mu_{s}}{\longrightarrow} Q_{s}
$$

where $r, s$ range over some countable set. Let $C_{r}, C_{s}$ be contexts obtained on these challenge transitions from the game of bisimulation up-to $\succeq_{\text {bis }}$ and context; assume that the appearance of a hole $[\cdot]_{i}$ in these contexts indicates that the hole is filled with the processes in the $i$-th pair of $\mathcal{R}$. For instance, if $P \stackrel{\mu_{r}}{\longrightarrow} P_{r}$ is the challenge transition, then by definition of bisimulation up-to $\succeq_{\text {bis }}$ and context, there are $Q^{\prime}$ and a context $C_{r}$ with $Q \stackrel{\widehat{\mu_{r}}}{\Longrightarrow} Q^{\prime}$ and $P_{r} \succeq_{\text {bis }} C_{r}[\widetilde{P}], Q^{\prime} \approx C_{r}[\widetilde{Q}]$.

Let $E_{r}, E_{s}$ be the expressions obtained from these contexts by replacing their hole $[\cdot]_{i}$ with $X_{i}$. Now, the contraction for the pair $P, Q$ is, assuming this is the pair $j$ in $\mathcal{R}$ :

$$
X_{j} \succeq \Sigma_{r} \mu_{r} . E_{r}+\Sigma_{s} \mu_{s} . E_{s}
$$

Now $\mathcal{R}_{1}$ and $\mathcal{R}_{2}$ are shown to be solutions of the resulting system of contraction reasoning as in the proof of Theorem 3.13.

For the second part of the theorem, let $\left\{X_{i} \succeq E_{i}\right\}_{i \in I}$ be the system of contractions of which $\widetilde{P}$ and $\widetilde{Q}$ are solutions. Suppose $P_{i} \stackrel{\mu}{\longrightarrow} P^{\prime}$. We have $P_{i} \succeq_{\text {bis }} E_{i}[\widetilde{P}]$, hence, by definition of bisimilarity contraction and since the contractions are weakly guarded, there are two subcases to consider:

1. $E_{i}[\widetilde{P}] \stackrel{\mu}{\longrightarrow} E_{i}^{\prime}[\widetilde{P}]$, for some $E_{i}^{\prime}$ with $P^{\prime} \succeq_{\text {bis }} E_{i}^{\prime}[\widetilde{P}]$;

2. $\mu=\tau$ and $P^{\prime} \succeq_{\text {bis }} E_{i}[\widetilde{P}]$. 
In (1), also $E_{i}[\widetilde{Q}] \stackrel{\mu}{\longrightarrow} E_{i}^{\prime}[\widetilde{Q}]$. It follows, from $Q \succeq_{\text {bis }} E_{i}[\widetilde{Q}]$, that $Q \stackrel{\widehat{\mu}}{\longrightarrow} Q^{\prime} \approx$ $E_{i}^{\prime}[\widetilde{Q}]$. This is sufficient, up to the context derived from $E_{i}^{\prime}$. In (2), we use $Q \Longrightarrow Q$ as matching transition, since $P^{\prime} \succeq_{\text {bis }} E_{i}[\widetilde{P}]$ and $Q \approx E_{i}[\widetilde{Q}]$, up to the context derived from $E$.

Remark 3.17 The observation in Remark 3.14 applies also to Theorem 3.16: the constructions in the theorem do not work with expansion in place of bisimilarity contraction. Indeed the definition of contraction was derived by attempts at obtaining theorems such as 3.13 and 3.16.

From Theorems 3.16 and 3.10 we derive:

Corollary 3.18 (soundness of 'bisimulation up-to $\succeq_{\text {bis }}$ and context'). If $\mathcal{R}$ is a bisimulation up-to $\succeq_{\text {bis }}$ and context, then $R \subseteq \approx$.

Having shown that the techniques of weakly-guarded contractions and 'bisimulation up-to $\succeq_{\text {bis }}$ and context' are equivalent, we can derive the soundness of one from the soundness of the other (in Corollary 3.18 we took the contraction technique as primitive). The complexity of the soundness proofs of the two techniques is similar. The main difference is that the expressions in the body of the contractions are weakly guarded, whereas the contexts of the 'up-to context' bisimulation techniques need not be so. As a consequence, in the proofs for the 'up-to context' techniques one has to reason about all possible interactions between a context and the processes plugged into it, proceeding by transition induction and a case analysis on the last rule used to derive a transition. With contractions this is avoided, exploiting the weak-guardedness condition and the unfolding of the contractions.

\subsection{Example: the lazy and eager servers}

We show a proof of the bisimilarity between the lazy and the eager systems of Section 2.3 using the technique of unique solution of contractions. This serves both as an an illustration of the application of the technique, and as a comparison with the technique based on the bisimulation proof method employed in the proof of Section 2.3.

The proof consists in showing that $\{L S\langle n\rangle\}_{n}$ and $\{E S\langle n\rangle\}_{n}$ are solutions of the following system of contractions:

$$
\left\{X_{n} \succeq c(z) .\left(X_{n+1} \mid R\langle c, n, z\rangle\right)\right\}_{n}
$$

We establish that $\{E S\langle n\rangle\}_{n}$ is a solution. For this we use simple algebraic laws: the expansion law, laws for pulling a process or a prefix outside of a restriction, and the laws

$$
\begin{aligned}
\boldsymbol{\nu} a(a(x) . P \mid \bar{a}\langle v\rangle . Q) & \sim \tau \cdot \boldsymbol{\nu} a(P\{v / x\} \mid Q) \\
\tau . P & \succeq_{\text {bis }} P
\end{aligned}
$$


These are essentially the laws used in proof in Section 2.3, using bisimulation up-to expansion and context. We thus have:

$$
\begin{aligned}
& E S\langle n\rangle \sim \boldsymbol{\nu} a(\tau \cdot(A\langle n+1\rangle \mid c(z) .(E \mid R\langle c, n, z\rangle))) \\
& \sim \quad \tau . c(z) .(\boldsymbol{\nu} a(A\langle n+1\rangle \mid E) \mid R\langle c, n, z\rangle) \\
& \succeq_{\text {bis }} \quad c(z) .(\boldsymbol{\nu} a(A\langle n+1\rangle \mid E) \mid R\langle c, n, z\rangle) \\
& =c(z) \cdot(E S\langle n+1\rangle \mid R\langle c, n, z\rangle)
\end{aligned}
$$

(The reader may want to compare this with the reasoning in Section 2.3 following the $\tau$ transition from $E S\langle n\rangle$.) We proceed similarly for $L S\langle n\rangle$.

The contraction (4) is not sequential, hence contractions and Theorem 3.10 cannot be replaced by equations and Theorem 2.8.

\section{Language generalisation}

We have shown the property of unique solution of weakly-guarded contractions in CCS. We generalise here the theorem to an arbitrary process language, using a more abstract condition. The generalisation serves both to better understand the validity of the theorem, and for applicability to languages that, unlike CCS, do not have an explicit prefixing construct.

For this generalisation we consider the case - standard in process algebra in which the syntax of the processes is the term algebra generated by some signature, and the semantics is given as an LTS. We call process language any such language. We use $\mathcal{L}$ to denote a generic process language, and $\mathcal{L}(\mathcal{X})$ for its extension with the contraction variables in $\mathcal{X}$.

Definition 4.1 A process language $\mathcal{L}$ is $\approx$-safe if, in $\mathcal{L}, \approx$ is a congruence relation, and its corresponding contraction $\succeq_{\text {bis }}$ is a precongruence.

For the results in this section, the condition on $\approx$ being a congruence could be weakened to $\approx$ being an equivalence.

In Theorem 3.10, the 'weakly guarded' hypothesis makes sure that the body of a contraction alone determines the first interaction. The body is thus autonomous: the interaction occurs without contributions from the terms that replace the contraction variables. Whenever the bodies of the contractions are autonomous in this sense, the unique-solution property holds.

Definition 4.2 (autonomous contractions) An expression $E$ of $\mathcal{L}(\mathcal{X})$ is autonomous if for all processes $\widetilde{P}$ of $\mathcal{L}$ we have:

- if $E[\widetilde{P}] \stackrel{\mu}{\longrightarrow} R$, then there is a context $C$ such that $R=C[\widetilde{P}]$, and for all $\widetilde{Q}$, also $E[\widetilde{Q}] \stackrel{\mu}{\longrightarrow} C[\widetilde{Q}]$.

A system of contractions $\left\{X_{i} \succeq E_{i}\right\}_{i \in I}$ is autonomous if each expression $E_{i}$ is autonomous.

We also need to make sure that the autonomy property is preserved is preserved underneath a context.

Definition 4.3 A process language $\mathcal{L}$ respects autonomy if for any context $C$ of $\mathcal{L}$ and for any autonomous expressions $\widetilde{E}$ of $\mathcal{L}(\mathcal{X})$, also the expression $C[\widetilde{E}]$ is autonomous. 
For the unique solution theorem, the crux is proving the analogous of Lemma 3.9.

Lemma 4.4 Suppose $\mathcal{L}$ is safe and respects autonomy, and $\widetilde{P}$ and $\widetilde{Q}$ are solutions for $\approx$ of a system of autonomous contractions. Then, for any context $C$, if $C[\widetilde{P}] \stackrel{\mu}{\Longrightarrow} R$, there is a context $C^{\prime}$ such that $R \succeq_{\text {bis }} C^{\prime}[\widetilde{P}]$ and $C[\widetilde{Q}] \stackrel{\widehat{\mu}}{\Longrightarrow} \approx C^{\prime}[\widetilde{Q}]$.

Proof Let $n$ be the length of the transition $C[\widetilde{P}] \stackrel{\mu}{\Longrightarrow} R$ (the number of 'strong steps' of which it is composed). We proceed by induction on $n$. If $n=0$ the assertion is trivial, for $R=C[\widetilde{P}]$ and $C^{\prime}=C$. Suppose the assertion holds for $n-1$; we treat the case $n$. Thus $C[\widetilde{P}] \stackrel{\mu}{\Longrightarrow} R$ can be written as $C[\widetilde{P}] \stackrel{\mu_{1}}{\longrightarrow} R^{\prime} \stackrel{\mu_{2}}{\Longrightarrow} R$ where one between $\mu_{1}$ and $\mu_{2}$ is $\mu$ and the other is a $\tau$ (they could also both be $\tau$ ). Let $C^{\prime \prime}[\widetilde{P}]$ and $C^{\prime \prime}[\widetilde{Q}]$ be the processes obtained from $C[\widetilde{P}]$ and $C[\widetilde{Q}]$ by unfolding the definitions of the contractions. Since the contractions are autonomous and the language respects autonomy, also $C^{\prime \prime}$ (thought as an expression) is autonomous.

Since both $\widetilde{P}$ and $\widetilde{Q}$ are solutions of the system of contractions, by the precongruence properties of $\succeq_{\text {bis }}$ we have $C[\widetilde{P}] \succeq_{\text {bis }} C^{\prime \prime}[\widetilde{P}]$ and $C[\widetilde{Q}] \succeq_{\text {bis }} C^{\prime \prime}[\widetilde{Q}]$. Applying the definition of $\succeq_{\text {bis }}$ on the transition $C[\widetilde{P}] \stackrel{\mu_{1}}{\longrightarrow} R^{\prime}$ we infer the existence of $C^{\prime \prime \prime}$ such that

$$
C^{\prime \prime}[\widetilde{P}] \stackrel{\widehat{\mu_{1}}}{\longrightarrow} C^{\prime \prime \prime}[\widetilde{P}] \preceq_{\text {bis }} R^{\prime}
$$

Since $C^{\prime \prime}$ is autonomous, also

$$
C^{\prime \prime}[\widetilde{Q}] \stackrel{\widehat{\mu_{1}}}{\longrightarrow} C^{\prime \prime \prime}[\widetilde{Q}]
$$

Moreover, from $R^{\prime} \stackrel{\mu_{2}}{\Longrightarrow} R$, we get $C^{\prime \prime \prime}[\widetilde{P}] \stackrel{\widehat{\mu_{2}}}{\Longrightarrow} \preceq_{\text {bis }} R$, with a transition $\widehat{\widehat{\Longrightarrow}}$ composed of no more than $n-1$ steps. We can therefore appeal to induction and infer the existence of $C^{\prime}$ with $R \succeq_{\text {bis }} C^{\prime}[\widetilde{P}]$ and $C^{\prime \prime \prime}[Q] \stackrel{\widehat{\mu_{2}}}{\Longrightarrow} \approx C^{\prime}[\widetilde{Q}]$.

Using this latter property, $C^{\prime \prime}[\widetilde{Q}] \stackrel{\widehat{\mu_{1}}}{\longrightarrow} C^{\prime \prime \prime}[\widetilde{Q}]$ and $C[\widetilde{Q}] \succeq_{\text {bis }} C^{\prime \prime}[\widetilde{Q}]$ we infer

$$
C[\widetilde{Q}] \stackrel{\widehat{\mu}}{\Longrightarrow} \approx C^{\prime}[\widetilde{Q}]
$$

This, together with $C[\widetilde{P}] \stackrel{\mu}{\Longrightarrow} R$ and $R \succeq_{\text {bis }} C^{\prime}[\widetilde{P}]$, concludes the proof.

Theorem 4.5 In a process language $\mathcal{L}$ that is safe and respects autonomy, a system of autonomous contractions has a unique solution for $\approx$.

The proof of the theorem is similar to that of Theorem 3.10, using Lemma 4.4 in place of Lemma 3.9.

Checking the autonomy property is often straightforward. For instance, in the case of the GSOS format [6], autonomy holds if, in the body $E$ of a contraction, all variables are underneath an axiom operator, that is, an operator that, as CCS prefix, is defined by means of SOS rules in which the set of hypothesis is empty. The preservation of autonomy underneath contexts is then straightforward.

Autonomy may not be preserved under a context if the language uses operators whose SOS rules make use of a lookahead, as it may happen with the 
tyft/tyxt format [14]. Technically, this means that an SOS rule may have two premises with a variable in the target of one being present in the source of the other premise. The following example shows that in this case autonomy may not be preserved and systems of autonomous contractions may not have a unique solution.

Example 4.6 Consider a process language with the following grammar

$$
P::=\mu . P|f(P)| \mathbf{0}
$$

where $\mu . P$ and $\mathbf{0}$ are the familiar CCS operators, and $f$ is defined by means of the following rule:

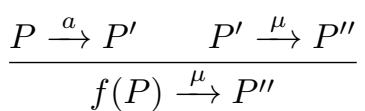

Now, the expression $a . f(X)$ is autonomous (for any $P$ we have $a . f(P) \stackrel{a}{\rightarrow} f(P)$ as the only transition); however $f(a . f(X))$ is not: when $X$ is instantiated with $a . b .0$ we can derive an $a$-transition, but this is not possible if $X$ is instantiated with a process unable to perform first $a$ and then $b$.

Furthermore, in the same language, the contraction

$$
X \succeq a . f(X)
$$

is autonomous, and yet both a.b. $\mathbf{0}$ and a.c. $\mathbf{0}$ are solutions.

The example above is the same used in [32] to show the problems of lookaheads in the techniques of 'bisimulation up-to'. We leave further comparisons for future work.

In some cases, autonomy may be better than the weakly-guarded hypothesis even if the calculus has a prefix operator: we shall see an example in Section 10, with the Higher-Order $\pi$-calculus, where autonomy allows us to capture occurrences of the contraction variables within output prefixes.

\section{Barbed congruence}

We (briefly) consider the application of the idea of contraction to barbed congruence [25], for various reasons. First, barbed congruence is a contextually-defined form of behavioural equivalence, and it paves the way to the treatment of other forms of contextual equivalence. Second, we want to show that - sometimes the contraction techniques make it possible to work directly with barbed congruence, even though it is contextually defined (e.g., the example with higher-order processes in Section 10). Third, the definition of barbed congruence applies to any language with a reduction semantics (i.e., a reduction relation and a barb, or observation, predicate), as opposed to the LTS semantics of the languages in earlier sections.

Thus the definitions and results in this section hold for any algebraic calculus (the term algebra over a signature) equipped with a reduction semantics, that is, a reduction relation $\longrightarrow$ and a barb predicate $\downarrow$. We use $\mathcal{R} \mathcal{L}$ for referring to a generic such language, and $\mathcal{R} \mathcal{L}(\mathcal{X})$ for its extension with the contraction variables in $\mathcal{X}$. (For CCS, $\longrightarrow$ is $\stackrel{\tau}{\longrightarrow}$ and $P \downarrow$ holds if $P \stackrel{\ell}{\rightarrow}$, for some visible 
action $\ell$.) As usual, $\Longrightarrow$ is the reflexive and transitive closure of $\longrightarrow$; and $P \Downarrow$ holds if there is $P^{\prime}$ with $P \Longrightarrow P^{\prime}$ and $P^{\prime} \downarrow$.

Definition 5.1 (barbed bisimulation and congruence) A relation $\mathcal{R}$ on the processes of $\mathcal{R} \mathcal{L}$ is a barbed bisimulation if whenever $P \mathcal{R} Q$ :

1. $P \longrightarrow P^{\prime}$ implies there is $Q^{\prime}$ such that $Q \Longrightarrow Q^{\prime}$ and $P^{\prime} \mathcal{R} Q^{\prime}$;

2. the converse, on the $\tau$-transitions emanating from $Q$, i.e., $Q \longrightarrow Q^{\prime}$ implies there is $P^{\prime}$ such that $P \Longrightarrow P^{\prime}$ and $P^{\prime} \mathcal{R} Q^{\prime}$;

3. if $P \downarrow$ then $Q \Downarrow$;

4. the converse, i.e., if $Q \downarrow$ then $P \Downarrow$.

Barbed bisimilarity, written $\approx_{\mathrm{bar}}$, is the union of all barbed bisimulations. Two processes $P$ and $Q$ are barbed congruent, written $P \approx_{\text {bar }}^{\mathrm{c}} Q$, if for each context $C$, it holds that $C[P] \approx_{\text {bar }} C[Q]$.

Remark 5.2 The definitions of barbed congruence in the literature often make use of a set of barb predicates; we use only one barb here for mere simplicity of presentation. A variant of barbed congruence is reduction-closed barbed congruence [16], in which the closure under contexts is placed within the definition of bisimilarity. The difference between the two variants has no consequences on the results in the paper.

In the 'contraction version' of barbed bisimilarity we write $Q \stackrel{\wedge}{\longrightarrow} Q^{\prime}$ if $Q \longrightarrow Q^{\prime}$ or $Q=Q^{\prime}$.

Definition 5.3 (barbed contraction, barbed congruence contraction). A relation $\mathcal{R}$ on the processes of $\mathcal{R} \mathcal{L}$ is a barbed contraction if, whenever $P \mathcal{R} Q$ :

1. $P \longrightarrow P^{\prime}$ implies there is $Q^{\prime}$ such that $Q \stackrel{\wedge}{\longrightarrow} Q^{\prime}$ and $P^{\prime} \mathcal{R} Q^{\prime}$;

2. $Q \longrightarrow Q^{\prime}$ implies there is $P^{\prime}$ such that $P \Longrightarrow P^{\prime}$ and $P^{\prime} \approx_{\text {bar }} Q^{\prime}$;

3. $P \downarrow$ implies $Q \downarrow$;

4. $Q \downarrow$ implies $P \Downarrow$.

Barbed contraction, written $\succeq_{\text {bar }}$, is the union of all barbed contractions. Barbed congruence contraction, written $\succeq_{\text {bar }}^{c}$, relates two processes $P$ and $Q$ if, for each context $C$, it holds that $C[P] \succeq_{\text {bar }} C[Q]$.

We transport the concept of autonomy to reduction-based semantics. A similar concept, called nondiscriminating context, has been used by Bonchi et al. [9].

Definition 5.4 (reduction-autonomous contractions) An expression $E$ of $\mathcal{R} \mathcal{L}(\mathcal{X})$ is reduction-autonomous if for all processes $\widetilde{P}$ and context $C$ of $\mathcal{R} \mathcal{L}$ :

- if $C[E[\widetilde{P}]] \longrightarrow R$, then there is a context $C^{\prime}$ such that $R=C^{\prime}[\widetilde{P}]$ and, for all $\widetilde{Q}$, also $C[E[\widetilde{Q}]] \longrightarrow C^{\prime}[\widetilde{Q}]$; 
- if $C[E[\widetilde{P}]] \downarrow$ then, for all $\widetilde{Q}$, also $C[E[\widetilde{Q}]] \downarrow$.

A system of contractions $\left\{X_{i} \succeq E_{i}\right\}_{i \in I}$ is reduction-autonomous if each expression $E_{i}$ is reduction-autonomous.

Barbed congruence and its contraction are, by definition, fully substitutive. Hence the safety requirement of Theorem 4.5 is not needed. In the property of unique solution for barbed congruence, the symbols $\asymp$ and $\succeq \asymp$ of Definition 3.5 become $\approx_{\text {bar }}^{\mathrm{c}}$ and $\succeq_{\mathrm{bar}}^{\mathrm{c}}$, respectively.

Theorem 5.5 In $\mathcal{R} \mathcal{L}$, any system of reduction-autonomous contractions has a unique solution for $\approx_{\text {bar }}^{\mathrm{c}}$.

Proof As for Theorems 3.10 and 4.5, we first prove something similar to Lemmas 3.9 and 4.4, namely:

Suppose $\widetilde{P}$ and $\widetilde{Q}$ are solutions for $\succeq_{\text {bar }}^{\mathrm{c}}$ to a system of reductionautonomous contractions. Then for any context $C$, if $C[\widetilde{P}] \Longrightarrow R$, there is a context $C^{\prime}$ such that $R \succeq_{\text {bar }} C^{\prime}[\widetilde{P}]$ and $C[Q] \stackrel{\widehat{\mu}}{\Longrightarrow} \approx_{\text {bar }}$ $C^{\prime}[\widetilde{Q}]$.

Using this fact, then one shows that, for $\widetilde{P}$ and $\widetilde{Q}$ solutions, the set of all pairs $(A, B)$ such that there is a context $C$ with $A \approx_{\text {bar }} C[\widetilde{P}]$ and $B \approx_{\text {bar }} C[\widetilde{Q}]$ is a barbed bisimilarity.

\section{Uniqueness of solution of contractions for non- coinductive equivalences}

We consider now non-coinductive equivalences. We focus on contextual equivalence [27] (i.e., may testing [11]), because it is widely studied. As the barbed congruence of Section 5, so contextual equivalence is contextually defined. Thus the setting considered is the same: an algebraic process language equipped with a reduction semantics. We reuse notations and terminologies from Section 5. Intuitively, two terms are contextually equivalent when they are equally observable, in any context.

Definition 6.1 (contextual equivalence) $P \approx_{\mathrm{ctx}} Q$ holds when $C[P] \Downarrow$ iff $C[Q] \Downarrow$, for all $C$.

The definition of the contextual equivalence contraction uses the predicates $P \Downarrow^{n}$, indicating that a barb is reached in $n$ steps (i.e., $P(\stackrel{\tau}{\longrightarrow})^{n} P^{\prime} \downarrow$, for some $\left.P^{\prime}\right)$.

Definition 6.2 (contextual equivalence contraction) $P \succeq_{\text {ctx }} Q$ if for all $C$ :

1. $C[P] \Downarrow^{n}$ implies $C[Q] \Downarrow^{m}$, for some $m \leq n$;

2. $C[Q] \Downarrow$ implies $C[P] \Downarrow$. 
Thus, referring to contextual equivalence, the symbols $\asymp$ and $\succeq \asymp$ of Definition 3.5 become $\approx_{\text {ctx }}$ and $\succeq_{\text {ctx }}$.

Theorem 6.3 A system of reduction-autonomous contractions has a unique solution for $\approx_{\text {ctx }}$.

Proof Suppose $\widetilde{P}$ and $\widetilde{Q}$ are solutions for $\succeq_{\text {ctx }}$ of a system of contractions $\left\{X_{i} \succeq E_{i}\right\}$, and consider a context $C$. We show that $C[\widetilde{P}] \Downarrow$ implies $C[\widetilde{Q}] \Downarrow$. Suppose $C[\widetilde{P}] \Downarrow^{n}$. We proceed by induction on $n$. First the case $n=0$, where $\Downarrow^{0}=\downarrow$.

Since $\widetilde{P} \succeq_{\text {ctx }} \widetilde{E}[\widetilde{P}]$, we have also $C[\widetilde{E}[\widetilde{P}]] \downarrow$ and, as $\widetilde{E}$ are reduction-autonomous, also $C[\widetilde{E}[\widetilde{Q}]] \downarrow$. Therefore, from $\widetilde{Q} \succeq_{\text {ctx }} \widetilde{E}[\widetilde{Q}]$, we derive $C[\widetilde{Q}] \Downarrow$.

Now the case $n>0$. Since $\widetilde{P} \succeq_{\text {ctx }} \widetilde{E}[\widetilde{P}]$, we have $C[\widetilde{E}[\widetilde{P}]] \Downarrow^{m}$ for some $m \leq n$. This means that either $C[\widetilde{E}[\widetilde{P}]] \downarrow$, or

$$
C[\widetilde{E}[\widetilde{P}]] \longrightarrow C^{\prime}[\widetilde{P}] \Downarrow^{m-1}
$$

(as $\widetilde{E}$ are reduction-autonomous, the processes used for the variables do not contribute to the reduction). If $C[\widetilde{E}[\widetilde{P}]] \downarrow$, we get $C[\widetilde{Q}] \Downarrow$, reasoning as above. Consider now (5). We also have $C[\widetilde{E}[\widetilde{Q}]] \longrightarrow C^{\prime}[\widetilde{Q}]$. By induction, from $C^{\prime}[\widetilde{P}] \Downarrow^{m-1}$ we infer $C^{\prime}[\widetilde{Q}] \Downarrow$. Therefore we have $C[\widetilde{E}[\widetilde{Q}]] \Downarrow$. From $\widetilde{Q} \succeq_{\text {ctx }} \widetilde{E}[\widetilde{Q}]$, we deduce $C[\widetilde{Q}] \Downarrow$.

Corollary 6.4 In CCS, a system of weakly-guarded contractions has a unique solution for $\approx_{\text {ctx }}$.

\subsection{Example: the lazy and eager servers, revisited}

Contextual equivalence does not have the congruence problems of bisimilarity for summation that motivated, in the presentation of CCS in Section 2, the use of guarded sums. We can therefore admit the full summation construct $\Sigma_{i} P_{i}$ in the grammar for the CCS processes. Such a flexibility will be useful in this example.

We revisit the lazy and eager servers in the example of Section 2.3. We modify the auxiliary server $A$, which was consulted by the main server before starting an interaction protocol with a client. In Section 2.3, the server was deterministic; now it is nondeterministic. Thus all definitions remain the same except that $A$ always returns an arbitrary integer:

$$
A \triangleq \Sigma_{n \in N} \quad \bar{a}\langle n\rangle . A
$$

The system with the lazy main server is now $L S \stackrel{\text { def }}{=} \boldsymbol{\nu} a(A \mid L)$, and the system with the eager main server is $E S \stackrel{\text { def }}{=} \boldsymbol{\nu} a(A \mid E)$, where $L$ and $E$ are as in Section 2.3. The timing difference between $L S$ and $E S$ in consulting $A$ is observable under bisimilarity. The reason is that an interaction

$$
E S \longrightarrow \boldsymbol{\nu} a(A \mid c(z) .(E \mid R\langle c, n, z\rangle)),
$$


in which $n$ is received from the auxiliary server $A$, is a commitment to using $n$ in the interaction with the next client. In contrast, $L S$ is unable to make such a commitment — its only initial transition is a visible one.

The difference is however not observable under contextual equivalence. We prove $L S \approx_{\text {ctx }} E S$ using the technique of unique solution of weakly-guarded contractions. The proof is similar to that with the deterministic auxiliary server and the bisimilarity contraction in Section 3.6, with a further simplification: a single contraction is sufficient, namely

$$
X \succeq c(z) . \Sigma_{n}(X \mid R\langle c, n, z\rangle)
$$

To show that both $L S$ and $E S$ are solutions for $\succeq_{\text {ctx }}$ of this contraction, we employ the same laws and algebraic reasoning of Section 3.6 (which are sound because bisimilarity implies contextual equivalence). An additional law is required in the proof of $E S$ :

$$
\Sigma_{i} \alpha . R_{i} \succeq_{\text {ctx }} \alpha . \Sigma_{i} R_{i} \quad(\alpha \text { is any prefix })
$$

(the law is actually valid for strong contextual equivalence, where two equal processes are required to reach an observable in the same number of steps). This is one of the most distinguishing laws of contextual equivalence. Using the laws we have:

$$
\begin{array}{rll}
E S=\boldsymbol{\nu} a(A \mid E) & \succeq_{c t x} & \Sigma_{n} \tau \cdot c(z) \cdot \boldsymbol{\nu} a(A|E| R\langle c, n, z\rangle) \\
& \succeq_{c t x} & \Sigma_{n} c(z) \cdot \boldsymbol{\nu} a(A|E| R\langle c, n, z\rangle) \\
& \succeq_{c t x} & c(z) \cdot \Sigma_{n}(\boldsymbol{\nu} a(A|E| R\langle c, n, z\rangle)) \\
& \succeq_{c t x} & c(z) \cdot \Sigma_{n}(\boldsymbol{\nu} a(A \mid E) \mid R\langle c, n, z\rangle) \\
= & c(z) \cdot \Sigma_{n}(E S \mid R\langle c, n, z\rangle)
\end{array}
$$

which shows that $E S$ is a solution of the contraction (6). The proof that also $L S$ is a solution is simpler.

The above proof is similar to the proofs with the servers in Sections 2.3 and 3.6. All these proofs, explicitly or implicitly, employ 'up-to context' reasoning; above the common context is $c(z) . \Sigma_{n}([\cdot] \mid R\langle c, n, z\rangle)$.

A proof that follows the definition of contextual equivalence would be hard due to the quantification on all contexts. In CCS, contextual equivalence coincides with trace equivalence. The equality in the example cannot be proved purely algebraically, using standard axiom systems for trace equivalence, because the systems compared are not finite or finite state (axiomatisations are complete only on these systems). One could show that $E S$ and $L S$ have the same traces proceeding by induction on the length of the traces. The proof is tedious, for instance because $R$ could be any process.

Remark 6.5 The proof of the equality between $E S$ and $L S$ reveals the essence of the technique based on unique solution of contractions. One employs some simple algebraic laws to prove that two tuples of terms are solutions of a certain system of contractions, from which the equality between the two tuples is derived from the unique-solution theorem. The algebraic laws may have been obtained in various ways (e.g., an axiomatisation of the equality for the finite terms). In our example we have used laws for bisimilarity, because it implies contextual 
equivalence, plus law (7). Law (7) is a well-known law in axiomatisations of trace equivalence; the law can also be easily proved directly in terms of contextual equivalence, reasoning by induction on the length on the number of $\tau$-steps needed to reach an observable.

\section{Trace equivalence}

In this section we briefly consider trace equivalence. In CCS and most process algebras, trace equivalence is a direct characterisation of contextual equivalence, in the same way as bisimilarity is for barbed congruence. Aside from this, the reason why we look at trace equivalence is that it is an example of a noncontextual and inductive behavioural equivalence. We use $s$ to range over traces, i.e., non-empty sequences of visible actions.

We assume to be in a generic process language $\mathcal{L}$ with an LTS semantics, as in Section 4. We write $P \stackrel{\mu}{\Longrightarrow}{ }_{n} P^{\prime}$ if $P \stackrel{\mu}{\Longrightarrow} P^{\prime}$ is derived using $n$ strong transitions (i.e., we have $P(\stackrel{\tau}{\longrightarrow})^{m} \stackrel{\mu}{\longrightarrow}(\stackrel{\tau}{\longrightarrow})^{m^{\prime}} P^{\prime}$ and $n=m+m^{\prime}+1$. If $s=\ell_{1}, \ldots, \ell_{n}$, then we write $P \stackrel{s}{\Longrightarrow}$ if $P \stackrel{\ell_{1}}{\Longrightarrow} P_{1} \stackrel{\ell_{2}}{\Longrightarrow} P_{2} \ldots P_{n-1} \stackrel{\ell_{n}}{\Longrightarrow} P_{n}$, for some processes $P_{1}, \ldots, P_{n}$. Similarly we write $P \stackrel{s}{\Rightarrow} m$ if there are $P_{1}, \ldots, P_{n}$ with $P \stackrel{\ell_{1}}{\Longrightarrow} m_{1} P_{1} \stackrel{\ell_{2}}{\Longrightarrow} m_{2} P_{2} \ldots P_{n-1} \stackrel{\ell_{n}}{\Longrightarrow} m_{n} P_{n}$, and $m=\Sigma_{i} m_{i}$.

Definition 7.1 Two processes $P, Q$ of $\mathcal{L}$ are trace equivalent, written $P \approx_{\operatorname{tr}} Q$, if for each trace $s$ we have $P \stackrel{s}{\Rightarrow}$ iff $Q \stackrel{s}{\Rightarrow}$.

Two processes $P, Q$ are in the trace equivalence contraction, written $P \succeq_{\operatorname{tr}} Q$, if, for each trace $s$ :

1. if $P \stackrel{s}{\Rightarrow}$ then $Q \stackrel{s}{\Rightarrow} m$ for some $m \leq n$;

2. if $Q \stackrel{s}{\Rightarrow}$ then $P \stackrel{s}{\Rightarrow}$.

A process language is $\approx_{\operatorname{tr}}$-safe if $\approx_{\operatorname{tr}}$ is a congruence and $\succeq_{\operatorname{tr}}$ a precongruence.

Theorem 7.2 In a process language $\mathcal{L}$ that is $\approx_{\operatorname{tr}}$-safe and respects autonomy, a system of autonomous contractions has a unique solution for $\approx_{\mathrm{tr}}$.

Refined forms of trace equivalence exist. For instance, ready trace equivalence $[4,13]$ combines traces with barbs. The idea of contraction and Theorem 7.2 can be adapted to ready traces by combining the treatment of traces in Definition 7.1 with the treatment of barbs in Definitions 5.3 and 6.2 .

\section{Non-applicability of the technique}

The contraction technique may be applied to any equivalence whose observables are finitary, in the sense that if an observable holds then it can be reached in a finite number of transitions. Bisimilarity is in this class: the observables are the weak transitions $\stackrel{\mu}{\Longrightarrow}$; each use of $\stackrel{\mu}{\Longrightarrow}$ is finitary because it is obtained by composing a finite number of strong transitions $(\stackrel{\tau}{\longrightarrow}$ and $\stackrel{\mu}{\longrightarrow})$. Different uses of $\stackrel{\mu}{\Longrightarrow}$ may have different lengths, but each length is finite. The same argument 
holds for $\Downarrow$, the observable of contextual equivalence. In all these cases, the contraction preorder precisely arises by playing with such finite measures.

There are behavioural equivalences, however, in which the observables are not finitary. For instance, an observable may be inherently coinductive, as for observables such as infinite traces and non-termination. We illustrate the possible failure of the contraction techniques in these cases using infinitary trace equivalence, whereby two processes are equated if they have the same traces, including the infinite ones. It is unclear how the contraction of infinitary trace equivalence should be defined. In any case, however, 'unique solution' would fail, even for guarded and sequential contractions. As an example, consider the processes $P \stackrel{\text { def }}{=} \Sigma_{n} a^{n}$ and $Q \stackrel{\text { def }}{=} P+a . ! a .0$. These processes are not infinitary trace equivalent. However, in an 'infinitary trace' semantics they both are solutions to the (guarded and sequential) contraction

$$
X \succeq a+a . X
$$

The definition of the the contraction for infinitary trace equivalence is irrelevant here, because the processes have no $\tau$-transitions. Similar problems arise for must equivalence, where non-termination is observable - the same counterexample of infinitary trace equivalence applies.

\section{Injecting contractions into the bisimulation game}

An advantage of bisimilarity, with respect to other behavioural equivalences, is the locality of the required checks: related states only have to match each other's immediate transitions. We can inject some locality also in other equivalences by introducing the corresponding contraction into a 'bisimulation up-to' game. We illustrate this possibility with contextual equivalence, which is inductive and contextual and therefore faraway from bisimilarity and its local checks. We consider the concrete case of the CCS language; thus in remainder of the section a process is meant to be a CCS process, and similarly for a relation.

Definition 9.1 (bisimulation up-to $\succeq_{\text {ctx }}$ ) A relation $\mathcal{R}$ is a bisimulation upto $\succeq_{\text {ctx }}$ if, whenever $P \mathcal{R} Q$, we have:

1. $P \stackrel{\mu}{\longrightarrow} P^{\prime}$ implies $Q \stackrel{\widehat{\mu}}{\Longrightarrow} Q^{\prime}$ and $P^{\prime} \succeq_{\text {ctx }} \mathcal{R} \approx_{\text {ctx }} Q^{\prime}$;

2. the converse of (1) on the actions from $Q$.

As in the case of ordinary bisimulation, bisimulation up-to $\succeq_{\text {ctx }}$ may be enhanced by combination with further up-to techniques. For instance, in the bisimulation up-to $\succeq_{\text {ctx }}$ and context the requirement $P^{\prime} \succeq_{\text {ctx }} \mathcal{R} \approx_{\text {ctx }} Q^{\prime}$ on the derivatives of Definition 2.3 becomes

$$
P^{\prime} \succeq_{\text {ctx }} \mathcal{R}^{\mathrm{c}} \approx_{\mathrm{ctx}} Q^{\prime}
$$

It is sufficient to analyse the most powerful technique ('up-to $\succeq_{\text {ctx }}$ and context'), and the results will also hold for the weaker 'up-to $\succeq_{\text {ctx }}$ '. We derive soundness from that of the corresponding contraction technique. 
Lemma 9.2 Suppose $\mathcal{R}$ is a bisimulation up-to $\succeq_{\text {ctx }}$ and context. Then there is a system of reduction-autonomous contractions, of the same size as $\mathcal{R}$, of which $\mathcal{R}_{1}$ and $\mathcal{R}_{2}$ are solutions for $\succeq_{\text {ctx }}$.

Proof The proof is similar to that of the first part of Theorem 3.16; we sketch below the main steps.

Suppose $\mathcal{R}$ is a bisimulation up-to $\succeq_{\text {ctx }}$ and context. We define the contractions satisfied by $\mathcal{R}_{1}$ and $\mathcal{R}_{2}$. Each pair in $\mathcal{R}$ gives rise to a contraction. We use the contraction variable $X_{i}$ for the contraction generated by the $i$-th pair in $\mathcal{R}$. In the solutions of the contractions, $X_{i}$ is replaced by the $i$-th process in $\mathcal{R}_{1}$ or $\mathcal{R}_{2}$. Consider $P \mathcal{R} Q$, and an enumeration of all the transitions from $P$ and $Q$

$$
P \stackrel{\mu_{r}}{\longrightarrow} P_{r}
$$

and

$$
Q \stackrel{\mu_{s}}{\longrightarrow} Q_{s}
$$

where $r, s$ range over some countable set. Let $C_{r}, C_{s}$ be contexts obtained on these challenge transitions from the game of bisimulation up-to $\succeq_{\text {ctx }}$ and context; assume that the appearance of a hole $[\cdot]_{i}$ in these contexts indicates that the hole is filled with the processes in the $i$-th pair of $\mathcal{R}$.

Let $E_{r}, E_{s}$ be the expressions obtained from these contexts by replacing their hole $[\cdot]_{i}$ with $X_{i}$. Now, the contraction for the pair $P, Q$ is, assuming this is the pair $j$ in $\mathcal{R}$ :

$$
X_{j} \succeq \Sigma_{r} \mu_{r} . E_{r}+\Sigma_{s} \mu_{s} . E_{s}
$$

The contractions are weakly guarded, which in CCS implies the reductionautonomous property.

A corollary of the lemma, and of the soundness of the unique-solution technique for reduction-autonomous contractions, is the soundness of the bisimulation technique.

Corollary 9.3 (soundness of bisimulation up-to $\succeq_{\text {ctx }}$ and context). Suppose a relation $\mathcal{R}$ is a bisimulation up-to $\succeq_{\text {ctx }}$ and context. Then $\mathcal{R} \subseteq \approx_{\text {ctx }}$.

Proof Follows from Theorem 6.3 and Lemma 9.2.

We have seen that, in the case of bisimilarity, the techniques of 'unique solution of contractions for $\approx$ ' and of 'bisimulation up-to contraction and context' are equivalent. For contextual equivalence, however, the former technique is more powerful. The reason is that, in the 'bisimulations up-to $\succeq_{\text {ctx }}$ and context' game, laws and equalities for $\approx_{\text {ctx }}$ are applied only after the derivatives of the processes in the pairs have been chosen. For instance, the lazy and eager servers of Section 6.1, $L S$ and $E S$, cannot be a pair of a bisimulation up-to $\succeq_{\text {ctx }}$ and context, for the same reason why they are not bisimilar: the challenge transition

$$
E S \stackrel{\tau}{\longrightarrow} \boldsymbol{\nu} a(A \mid c(z) .(E \mid R\langle c, n, z\rangle))
$$

cannot be matched by $L S$.

In some cases, however, the obstacle can be bypassed. We show this for the processes $L S$ and $E S$ of the server example. We relate $L S$ to a contraction 
$E S^{\prime}$ of $E S$, obtained by abstracting the initial private communication with the auxiliary server $A$ :

$$
E S \succeq_{\text {ctx }} \Sigma_{n} \boldsymbol{\nu} a(A \mid c(z) .(E \mid R\langle c, n, z\rangle)) \stackrel{\text { def }}{=} E S^{\prime}
$$

Now, the singleton relation $\left\{\left(L S, E S^{\prime}\right)\right\}$ is a bisimulation up-to $\succeq_{\text {ctx }}$ and context. The processes in the pair initially may only perform an input at $c$; if $v$ is the value received in the input, then the transitions are

$$
\begin{array}{cccc} 
& E S^{\prime} & \stackrel{c\langle v\rangle}{\longrightarrow} & \Sigma_{n} \boldsymbol{\nu} a(A|E| R\langle c, n, v\rangle) \stackrel{\text { def }}{=} E S_{1}^{\prime} \\
\text { and } \quad L S & \stackrel{\nu}{=} \boldsymbol{\nu} a(A \mid c(z) \cdot a(x) \cdot(L \mid R\langle c, x, z\rangle)) \\
& \stackrel{c\langle v\rangle}{\longrightarrow} & \boldsymbol{\nu} a(A \mid a(x) .(L \mid R\langle c, x, v\rangle)) \stackrel{\text { def }}{=} L S_{1}
\end{array}
$$

Now we have, using algebraic reasoning similar to that in previous examples with the servers and (8):

$$
\begin{array}{ccc}
E S_{1}^{\prime} & \sim & \Sigma_{n}(\boldsymbol{\nu} a(A \mid E) \mid R\langle c, n, v\rangle) \\
& \succeq_{\text {ctx }} & \Sigma_{n}\left(E S^{\prime} \mid R\langle c, n, v\rangle\right) \\
\text { and } \quad L S_{1} & \succeq_{\text {ctx }} & \Sigma_{n}(\boldsymbol{\nu} a(A \mid L) \mid R\langle c, x, v\rangle) \\
& = & \Sigma_{n}(L S \mid R\langle c, x, v\rangle)
\end{array}
$$

This is sufficient, up-to $\succeq_{\text {ctx }}$ and context. Finally, having proved $E S \approx_{\text {ctx }} E S^{\prime}$ and $E S^{\prime} \approx_{\mathrm{ctx}} L S$, we derive $E S \approx_{\mathrm{ctx}} L S$ by transitivity.

Other behavioural equivalences and their contractions can be injected into the 'bisimulation up to' game, along the lines of what is done here for contextual equivalence.

\section{Higher-order languages}

The contraction technique may also be used in higher-order languages such as the $\lambda$-calculus and the Higher-Order $\pi$-calculus [41]. We refrain from attempting to produce a general theory of contractions for higher-order languages, comparable in power to the bisimulation proof method for these languages. We leave this for future work. (For instance, in higher-order languages bisimilarity is usually rather different in shape to the standard bisimilarity of Definition 2.1, and this should have a considerable impact on contractions and their proofs.) Here we simply show that the transport to a higher-order setting of the most basic contraction techniques - those involving a reduction semantics and contextually-defined equivalences - can still be useful. We illustrate this on the Higher-Order $\pi$-calculus. We consider the Higher-Order $\pi$-calculus in its simplest form, where only processes can be communicated. Below is the syntax.

$$
\begin{aligned}
& P::=\bar{a}\langle P\rangle \cdot Q \text { output prefix } \\
& a(x) . P \text { input prefix } \\
& x \quad \text { process variable } \\
& \text { עa } P \text { restriction } \\
& P \mid Q \quad \text { parallel composition } \\
& \text { o nil }
\end{aligned}
$$


Structural congruence: the least congruence $\equiv$ such that

- $P|Q \equiv Q| P, P|(Q \mid R) \equiv(P \mid Q)| R, P \mid \mathbf{0} \equiv P ; \boldsymbol{\nu} a \mathbf{0} \equiv \mathbf{0}$, $\boldsymbol{\nu} a \boldsymbol{\nu} b P \equiv \boldsymbol{\nu} b \boldsymbol{\nu} a P ;(\boldsymbol{\nu} a P) \mid Q \equiv \boldsymbol{\nu} a(P \mid Q)$, if $a$ not free in $Q$

The reduction relation $P \longrightarrow Q$ is the least relation such that:

$$
\begin{gathered}
a(x) . R|\bar{a}\langle P\rangle . Q \longrightarrow R\{P / x\}| Q \quad \frac{P \longrightarrow P^{\prime}}{P\left|Q \longrightarrow P^{\prime}\right| Q} \quad \frac{P \longrightarrow P^{\prime}}{\nu a P \longrightarrow \nu a P^{\prime}} \\
\frac{P \equiv P^{\prime} \longrightarrow P^{\prime \prime} \equiv P^{\prime \prime \prime}}{P \longrightarrow P^{\prime \prime \prime \prime}}
\end{gathered}
$$

Figure 2: The reduction semantics for $\mathrm{HO} \pi$

The reduction semantics is standard. It uses a structural congruence that permits the rearrangement of parallel compositions and restrictions so that the participants in a potential communication can be brought into immediate proximity; and a reduction relation that describes the act of communication itself. The rules are reported in Figure 2.

We use $a, b, c, \ldots$ to range over names, and $x, y, z, \ldots$ to range over variables; we call them language variables (to distinguish them from the contraction or equation variables such as $X, Y)$. An input $a(x) . P$ binds the free occurrences of variable $x$ in $P$; similarly a restriction $\boldsymbol{\nu}$ a $P$ binds the free occurrences of name $a$ in $P$. A term is open or closed depending on whether it may, or may not, have free language variables (in any case, it may have free names).

Systems of contractions in the Higher-Order $\pi$-calculus In the definition of barbed congruence and its contraction, the only technicality of higherorder languages that has to be taken into account is the distinction between open and closed terms. This is dealt with in the expected manner. All running terms are supposed to be closed. Thus the definitions of equivalences and preorders in earlier sections (e.g., barbed congruence and its contraction) are meant to be on closed terms. The definitions are generalised to open expressions by requiring instantiation of the language variables with all closing substitutions, i.e., substitutions that make the terms closed (using, in contextual definitions, closing contexts rather than closing substitutions would yield the same relations).

An example The example is about two ways of modeling the replication operator. We consider the equality (barbed congruence) between the terms $\bar{c}\langle A\rangle$ and $\bar{c}\langle B\rangle$, where

$$
A \stackrel{\text { def }}{=} b(y) . \nu a(M \mid \bar{a}\langle M\rangle) \quad \text { for } M \stackrel{\text { def }}{=} a(x) .(y|x| \bar{a}\langle x\rangle)
$$

and

$$
B \stackrel{\text { def }}{=} b(y) . \nu a(N \mid \bar{a}\langle y \mid N\rangle) \quad \text { for } N \stackrel{\text { def }}{=} a(x) .(x \mid \bar{a}\langle x\rangle) .
$$

Terms $\bar{c}\langle A\rangle$ and $\bar{c}\langle B\rangle$ send on $c$ processes $(A$ and $B)$ that can receive a process at $b$ and then replicate this process. Indeed, if $P$ is the process so received, 
assuming $a$ does not occur free in $P$, in one case we obtain the term

$$
A_{P} \stackrel{\text { def }}{=} \boldsymbol{\nu} a(M\{P / y\} \mid \bar{a}\langle M\{P / y\}\rangle)
$$

and in the other case

$$
B_{P} \stackrel{\text { def }}{=} \boldsymbol{\nu} a(N \mid \bar{a}\langle P \mid N\rangle),
$$

and then we have:

$$
\begin{aligned}
& A_{P} \longrightarrow P\left|A_{P} \longrightarrow P\right| P \mid A_{P} \longrightarrow \cdots \\
& B_{P} \longrightarrow P\left|B_{P} \longrightarrow P\right| P \mid B_{P} \longrightarrow \cdots
\end{aligned}
$$

The internal structure of $A_{P}$ and $B_{P}$ is however different.

A system of contractions that proves $\bar{c}\langle A\rangle \approx_{\mathrm{bar}}^{\mathrm{c}} \bar{c}\langle B\rangle$ is the following:

$$
\begin{aligned}
& X \succeq \bar{c}\langle Y\rangle . \mathbf{0} \\
& Y \succeq b(y) . Z \\
& Z \succeq \tau .(y \mid Z)
\end{aligned}
$$

These contractions are reduction-autonomous, and therefore have a unique solution for barbed congruence. Note that, in the first contraction, a contraction variable occurs within the initial output prefix. Thus the contraction is not weakly guarded. Still, the contraction is reduction-autonomous because a process that replaces the variable (and that therefore represents the value emitted in the output) does not contribute to the first action. Note also that the third contraction is open - it has $y$ as a free variable. Two solutions for $\succeq_{\text {bar }}^{c}$ (the barbed congruence contraction) to the above system of three contractions are, respectively:

1. $\bar{c}\langle A\rangle, A$, and $\boldsymbol{\nu} a(M \mid \bar{a}\langle M\rangle)$;

2. $\bar{c}\langle B\rangle, B$, and $\boldsymbol{\nu} a(N \mid \bar{a}\langle y \mid N\rangle)$.

The third process of each solution has $y$ free, as its corresponding contraction. To prove that these are solutions, we need a few simple algebraic laws. such as

- $\boldsymbol{\nu} a(\bar{a}\langle R\rangle \cdot P \mid a(x) \cdot Q) \succeq_{\mathrm{bar}}^{\mathrm{c}} \boldsymbol{\nu} a(P \mid Q\{R / x\})$,

and laws that modify the scope of a restriction.

Using the bisimulation proof method, the proof of the equality between $\bar{c}\langle A\rangle$ and $\bar{c}\langle B\rangle$ is more cumbersome; with the bisimulation techniques currently available, a proof requires an infinite relation. Even in the case of environmental bisimulation, where a form of 'up-to context' is available, the relation used in [42] for the same example is infinite because, intuitively, the values emitted, $A$ and $B$, have to be stored in an environment, and can then be played back at any time, possibly several times. What makes the difference is that contractions here allow us to extract a common context that incorporates the prefix for the initial action (cf. the contraction for the variable $X$ ). In contrast, in 'up-to context' techniques for bisimulation, contexts are only removed from the derivatives, after firing an initial prefix. 


\section{Further related work}

Milner's theorem about unique solution of equations stems from an axiomatisation of bisimulation on finite-state processes [26]. Indeed, in axiomatisations of behavioural equivalences $[24,3]$, the corresponding rule plays a key role and is called fixed-point rule, or recursive specification principle; see also [33], for trace equivalence. The possible shapes of the solutions of systems of equations, in connection with conditions on the guardedness of the equations, is studied by Baeten and Luttik [5]; the setting, however, in contrast with our paper, is that of strong behavioural equivalences.

Unique solution of equations has been considered in various settings, including languages, algebraic power series and pushdown automata (see the surveys $[20,29]$ ), as well as in coalgebras (e.g., [23]). These models, however, may not have the analogous of 'internal step', around which all the theory of contractions is built. In functional languages, unique solution of equations is sometimes called 'unique fixed-point induction principle'. See for instance [39], in which the conditions resembles Milner's conditions of Theorem 2.8, and [18], which studies equations on streams advocating a condition based on the notion of 'contractive function' (the word 'contraction' here is unrelated to its use in our paper). In automata theory and formal languages, Arden's Rule (or Lemma) [1] is widely used for deriving the languages accepted by automata and manipulating regular expressions via solutions to equations. Conditions on the empty word are needed to guarantee guardedness of the equations and hence unicity of a solution (one may see similarities between the empty word of automata and the $\tau$ actions of processes, see the discussion in the next section).

A tutorial on bisimulation enhancements is [32]. 'Up-to context' techniques have been formalised in a coalgebraic setting, and adapted to languages whose LTS semantics adheres to the GSOS format [6]; see for instance [7], which uses lambda-bialgebras, a generalisation of GSOS to the categorical framework.

The techniques in Section 9, transporting the bisimulation proof method and some of its enhancements onto non-coinductive equivalences, remind us of techniques for reducing non-coinductive equivalences to bisimilarity. For instance, trace equivalence on nondeterministic processes can be reduced to bisimilarity on deterministic processes, following the powerset construction for automata [17]; a similar reduction can be made for testing equivalence [10]. These results rely on transformations of transitions systems, which modify the nondeterminism and the set of states, in such a way that a given equivalence on the original systems corresponds to bisimilarity on the altered systems. In contrast, in the techniques of Section 9 the transformation of processes is performed dynamically, alongside the bisimulation game: two processes are manipulated only when necessary, i.e., when their immediate transitions would break the bisimulation game.

In CSP [15], some beautiful results have been obtained in which systems of equations have unique solutions provided their least fixed point (intuitively obtained by infinite unfolding of the equations) does not contain divergent states; see $[35,36]$. In CSP the semantics has usually a denotational flavour and, most important, the reference behavioural equivalence, failure equivalence, is divergent sensitive. As mentioned in Section 8, currently we do not know how to handle divergence in the theory of contractions, as divergence is not a finitary observable. We note however that (at least in the equivalences considered in the paper) unique solution of contractions holds in cases where the infinite un- 
folding of the contractions would introduce divergence: e.g., the contractions of Example 3.11, as well as the contractions employed in the examples about the lazy and eager servers (where divergence may appear if, in the interaction protocol with a client, the main server is called back).

\section{Conclusions and future work}

In this paper we have presented operational techniques, based on the idea of contraction, for proving weak behavioural equivalences, that is, equivalences that abstract from internal moves. We have focused on concurrent languages but the techniques are not meant to be specific to concurrency. We have illustrated the techniques with bisimulation, the most natural ground of application, discussing also completeness. We have then shown that the technique of unique solution of contractions can be transported onto other equivalences, with finitary observables (e.g., contextual equivalence, barbed congruence, trace equivalence). We have also seen that the contraction preorders can be injected into the bisimulation game. In the case of bisimulation, this leads to a (minor) improvement of an existing technique, namely 'bisimulation up-to expansion and context'. The case of non-coinductive or contextual equivalences such as contextual equivalence is more interesting: we can use the bisimulation proof method (enhanced with up-to context) for reasoning on these equivalences, combined with algebraic laws for manipulating states whose immediate transitions would break the bisimulation game. Such techniques allow us, implicitly, to transfer 'up-to context' forms of reasoning, originally proposed for labeled bisimilarities and their proof method, onto equivalences that are contextual or non-coinductive.

As for the technique based on equations, so the technique based on contractions is meant to be used in combination with algebraic reasoning, on terms whose behaviour is not finite or finite-state: the recursion on the contraction variables captures the infinite behaviour of terms, and the proof that certain processes are solutions is carried out with pure algebraic reasoning.

In comparison with equations, a drawback of unique solution of contractions for an equivalence $\asymp$ is that the solutions are not $\asymp$-interchangeable: it may be that $P$ is solution and $Q$ is not, even though $P \asymp Q$.

The proof of completeness of the 'unique solution of contractions' method with respect to the bisimulation proof method uses the sum operator to express the possible initial actions of a process. We would like to see how completeness can be recovered in languages in which the sum operator is missing. One may consider the introduction of an operator akin to sum, to be used only for writing contractions. Also, we did not tackle completeness in equivalences other than bisimilarity.

We have related the contraction technique to bisimulation enhancements such as 'up-to expansion and context'. While powerful, these are not the only possible enhancements. It would be interesting to see whether other enhancements can be captured using contractions or similar notions.

We would like to understand on which behavioural equivalences the technique of unique solution of contractions works. We mentioned in Section 8 that it seems to work if the observables of the equivalence are finitary. More experimentation is needed to clarify this point, and formalise appropriate conditions. A first candidate for further experiments could be fair-must testing [28, 34]. 
In the example with a higher-order language, we have applied the most basic contraction techniques - those for contextually-defined equivalences. The use of other contraction techniques requires further investigation. Such study may shed light on the applicability of up-to context techniques to higher-order languages. In a higher-order language, while there are well-developed techniques for proving that a bisimulation is a congruence [31], up-to context is still poorly understood $[22,21,19,42,30]$. For instance, for pure $\lambda$-calculi and applicative bisimilarity, the soundness of the full up-to context technique (allowing one to remove any context, possibly binding variables of the enclosed terms) still represents an open problem.

Another setting in which up-to context techniques have been recently applied is that of language equivalence for automata, see e.g., [8, 37]. The technique we have developed in this paper are for languages with internal moves. In the case of automata, a $\tau$-action could correspond to the empty word, which is absorbed in concatenations of words, in the same way as $\tau$-actions are absorbed in concatenation of traces. However, taking into account the way the empty word (or the empty language) and $\tau$-steps are used, the analogy seems light. It is unclear whether contractions could be useful on automata.

Our original motivation for studying contractions was to better understand 'up-to context' enhancements of the bisimulation proof method and their soundness. More broadly, the goal of the line of work reported is to improve our understanding of bisimilarity and the proof techniques for it, including the possibility of exporting the techniques onto other equivalences.

\section{Acknowledgments}

I have benefited from discussions with Luca Aceto, Jos Baeten, Filippo Bonchi, Matthew Hennessy, Bill Roscoe, David Sands, and from the comments of the anonymous referees. This work has been partially supported by the ANR project 12IS02001 'PACE', and the MIUR-PRIN project 'CINA'.

\section{References}

[1] D.Ñ.Ârden. Delayed logic and finite state machines. Theory of Computing Machine Design, 1-35, Univ. of Michigan Press, USA, 1960.

[2] S. Arun-Kumar and M. Hennessy. An efficiency preorder for processes. Acta Informatica, 29:737-760, 1992.

[3] J.C.M. Baeten, T. Basten, and M.A. Reniers. Process Algebra: Equational Theories of Communicating Processes. Cambridge University Press, 2010.

[4] Jos C. M. Baeten, Jan A. Bergstra, and Jan Willem Klop. Ready-trace semantics for concrete process algebra with the priority operator. Comput. J., 30(6):498-506, 1987.

[5] Jos C. M. Baeten and Bas Luttik. Unguardedness mostly means many solutions. Theor. Comput. Sci., 412(28):3090-3100, 2011.

[6] B. Bloom, S. Istrail, and A.R. Meyer. Bisimulation can't be traced. Journal of the ACM, 42(1):232-268, 1995. 
[7] Filippo Bonchi, Daniela Petrisan, Damien Pous, and Jurriaan Rot. Coinduction up to in a fibrational setting. Proc. LICS'14, to appear., 2014.

[8] Filippo Bonchi and Damien Pous. Checking nfa equivalence with bisimulations up to congruence. In Roberto Giacobazzi and Radhia Cousot, editors, Proc. POPL'13, pages 457-468. ACM, 2013.

[9] Filippo Bonchi, Fabio Gadducci, and Giacoma V. Monreale A General Theory of Barbs, Contexts, and Labels ACM Trans. Comput. Log., 15(4):35:1$35: 27,2014$.

[10] Rance Cleaveland and Matthew Hennessy. Testing equivalence as a bisimulation equivalence. Formal Asp. Comput., 5(1):1-20, 1993.

[11] R. De Nicola and R. Hennessy. Testing equivalences for processes. Theoretical Computer Science, 34:83-133, 1984.

[12] R.J. van Glabbeek. The linear time-branching time spectrum II (the semantics of sequential systems with silent moves). In E. Best, editor, Proc. CONCUR '93, volume 715. Springer Verlag, 1993.

[13] R.J. van Glabbeek. The linear time-branching time spectrum I. In A. Ponse J. Bergstra and S. Smolka, editors, Handbook of Process Algebra, pages 3-99. Elsevier, 2001.

[14] J.F. Groote and F.W. Vaandrager. Structured operational semantics and bisimulation as a congruence. Information and Computation, 100:202-260, 1992.

[15] C.A.R. Hoare. Communicating Sequential Processes. Prentice Hall, 1985.

[16] K. Honda and N. Yoshida. On reduction-based process semantics. Theoretical Computer Science, 152(2):437-486, 1995.

[17] John E. Hopcroft, Rajeev Motwani, and Jeffrey D. Ullman. Introduction to Automata Theory, Languages, and Computation (3rd Edition). AddisonWesley, Boston, MA, USA, 2006.

[18] G. Hutton and M. Jaskelioff. Representing Contractive Functions on Streams. Submitted, 2011.

[19] Vassileios Koutavas and Mitchell Wand. Small bisimulations for reasoning about higher-order imperative programs. In Proceedings of the 33rd ACM SIGPLAN-SIGACT Symposium on Principles of Programming Languages, pages $141-152,2006$.

[20] Michal Kunc. Simple language equations. Bulletin of the EATCS, 85:81102, 2005.

[21] S.B. Lassen. Relational reasoning about contexts. In Higher-order operational techniques in semantics, pages 91-135. Cambridge University Press, 1998.

[22] S.B. Lassen. Bisimulation in untyped lambda calculus: Böhm trees and bisimulation up to context. Electr. Notes Theor. Comput. Sci., 20:346374, 1999. 
[23] S. Milius, L.S. Moss, and D. Schwencke. Abstract GSOS rules and a modular treatment of recursive definitions. Logical Methods in Computer Science, $9(3), 2013$.

[24] R. Milner. Communication and Concurrency. Prentice Hall, 1989.

[25] R. Milner and D. Sangiorgi. Barbed bisimulation. In W. Kuich, editor, Proc. 19th ICALP, volume 623 of Lecture Notes in Computer Science, pages 685-695. Springer Verlag, 1992.

[26] Robin Milner. A complete axiomatisation for observational congruence of finite-state behaviors. Inf. Comput., 81(2):227-247, 1989.

[27] James H. Morris. Lambda-Calculus Models of Programming Languages. Phd thesis MAC-TR-57, M.I.T., project MAC, Dec. 1968.

[28] V. Natarajan and Rance Cleaveland. Divergence and fair testing. In Proceedings of ICALP'95, volume 944 of Lecture Notes in Computer Science, pages 648-659. Springer Verlag, 1995.

[29] Ion Petre and Arto Salomaa. Algebraic systems and pushdown automata. In Handbook of Weighted Automata, EATCS Series, pages 257-289. Springer, 2009.

[30] Adrien Piérard and Eijiro Sumii. Sound bisimulations for higher-order distributed process calculus. In Martin Hofmann, editor, Proc. FOSSACS, volume 6604 of Lecture Notes in Computer Science, pages 123-137. Springer, 2011.

[31] Andrew Pitts. Howe's method. In Davide Sangiorgi and Jan Rutten, editors, Advanced Topics in Bisimulation and Coinduction. Cambridge University Press, 2012.

[32] Damien Pous and Davide Sangiorgi. Enhancements of the bisimulation proof method. In Davide Sangiorgi and Jan Rutten, editors, Advanced Topics in Bisimulation and Coinduction. Cambridge University Press, 2012.

[33] Alexander Moshe Rabinovich. A complete axiomatisation for trace congruence of finite state behaviors. In Stephen D. Brookes, Michael G. Main, Austin Melton, Michael W. Mislove, and David A. Schmidt, editors, Proc. 9th MFPS, volume 802 of Lecture Notes in Computer Science, pages 530543. Springer, 1993.

[34] Arend Rensink and Walter Volger. Fair testing. Information and Computation, 205:125-198, 2007.

[35] A. W. Roscoe. The theory and practice of concurrency. Prentice Hall, 1998.

[36] A. W. Roscoe. Understanding Concurrent Systems. Springer, 2010.

[37] Jurriaan Rot, Marcello M. Bonsangue, and Jan J. M. M. Rutten. Coinductive proof techniques for language equivalence. In Adrian Horia Dediu, Carlos Martín-Vide, and Bianca Truthe, editors, Proc. LATA, volume 7810 of Lecture Notes in Computer Science, pages 480-492. Springer, 2013. 
[38] D. Sangiorgi. Locality and true-concurrency in calculi for mobile processes. In TACS'94, volume 789 of Lecture Notes in Computer Science, pages 405424. Springer, 1994.

[39] David Sands. Computing with Contexts: A simple approach. ENTCS, volume 10, Elsevier, 1998

[40] D. Sangiorgi and R. Milner. The problem of "Weak Bisimulation up to". In W.R. Cleveland, editor, Proc. CONCUR '92, volume 630 of Lecture Notes in Computer Science, pages 32-46. Springer Verlag, 1992.

[41] D. Sangiorgi and D. Walker. The $\pi$-calculus: a Theory of Mobile Processes. Cambridge University Press, 2001.

[42] Davide Sangiorgi, Naoki Kobayashi, and Eijiro Sumii. Environmental bisimulations for higher-order languages. ACM Trans. Program. Lang. Syst., 33(1):5, 2011. 